Article

\title{
On Optimal Fuzzy Best Proximity Coincidence Points of Proximal Contractions Involving Cyclic Mappings in Non-Archimedean Fuzzy Metric Spaces
}

\author{
Manuel De la Sen ${ }^{1, *}$, Mujahid Abbas ${ }^{2,3}$ and Naeem Saleem ${ }^{4,5}$ \\ 1 Institute of Research and Development of Processes IIDP, Faculty of Science and Technology, \\ University of the Basque Country, P.O. Box 644 de Bilbao, Barrio Sarriena, 48940 Leioa (Bizkaia), Spain \\ 2 Department of Mathematics, King Abdulaziz University, P.O. Box 80203, Jeddah 21589, Saudi Arabia; \\ mujahid.abbas@up.ac.za \\ 3 Department of Mathematics, Government College University, 54000 Lahore, Pakistan \\ 4 Department of Mathematics, National University of Computer and Emerging Sciences, Milaad Street, \\ Lahore 54000, Pakistan; naeem.saleem@umt.edu.pk or naeem.saleem2@gmail.com \\ 5 Department of Mathematics, University of Management and Technology, C-II Johar Town, \\ Lahore 54000, Pakistan \\ * Correspondence: manuel.delasen@ehu.es; Tel.: +34-946-012-548
}

Academic Editor: Pasquale Vetro

Received: 30 March 2016; Accepted: 27 March 2017; Published: 1 April 2017

\begin{abstract}
The main objective of this paper is to deal with some properties of interest in two types of fuzzy ordered proximal contractions of cyclic self-mappings $T$ integrated in a pair $(g, T)$ of mappings. In particular, $g$ is a non-contractive fuzzy self-mapping, in the framework of non-Archimedean ordered fuzzy complete metric spaces and $T$ is a $p$-cyclic proximal contraction. Two types of such contractions (so called of type I and of type II) are dealt with. In particular, the existence, uniqueness and limit properties for sequences to optimal fuzzy best proximity coincidence points are investigated for such pairs of mappings.
\end{abstract}

Keywords: fixed points; best proximity points; fuzzy set; fuzzy metric; optimal fuzzy best proximity coincidence points; proximal $\psi$-contractions of types I and II

\section{Introduction}

Concepts and related results on fuzzy sets in several research disciplines are abundant in the background literature. From a mathematical context, studies are available, for instance, in [1-18] and the references therein, following its introduction and characterization by Zadeh [4]. Among the research performed on the subject, effort has been devoted to the investigation of the existence and uniqueness of fixed points, best proximity points, fuzzy fixed points, fuzzy best proximity points, common fuzzy fixed points and optimal fuzzy coincidence points [15-28]. Also, research has been devoted to related properties of convergence of sequences to the abovementioned relevant points. Fixed Point Theory is also relevant to the stability properties of some iterative schemes of that of dynamic systems [29-33], as an alternative tool to other classical techniques like Lyapunov stability. (See, for instance, [33-37].) There are also abundant studies on all such topics in classical metric spaces and Banach spaces, either in the fuzzy formalism or not necessarily under the fuzzy formalism, including a lot of research on contractive and non-expansive mappings, self-mappings and, in particular, cyclic proximal mappings. (See, for instance, [26-29,38-43] and the references therein concerning different iterative schemes and their relations to proximal split feasibility, variational inequalities and fixed point problems. There are also recent studies on the generalizations of several types of contractions in [31] with an introduction of the so-called simulation function. 
Recent work in fuzzy metric spaces and probabilistic metric spaces can be found in [22-25]. Also, the so-called simulation function is introduced and discussed in [31] related to a new special generalized contraction that generalizes the Banach contraction and unifies several previously known types of contractions.

There are certain real-life problems for which fixed points, best proximity points, optimal coincidence points or optimal best proximity coincidence points do not exist, so their approximate counterparts are looked for in order to have an approximate solution of the problem at hand. We recall the following basic concepts:

If $(X, d)$ is a metric space, $A, B \subseteq X$ are non-empty then:

(1) $x \in A$ is a fixed point of $T: A \rightarrow A$ if $d(x, T x)=0$;

(2) $x \in A$ is an approximate fixed point of $T: A \rightarrow A$ if $d(x, T x)=\inf \{d(y, T x): y \in A\}$;

(3) $x \in A$ is a best proximity point of $T: A \rightarrow B$ in $A$ if $d(x, T x)=d(A, B)=$ inf $\{d(z, y): z \in A, y \in B\}$;

(4) $x \in A$ is an approximate best proximity point of $T: A \rightarrow B$ in $A$ if $d(x, T x)=$ $\inf \{d(y, T x): y \in A\}$;

Note that a fixed point of $T: A \rightarrow A$ is an approximate fixed point of $T: A \rightarrow A$ while the converse is not true, in general. Also, a best proximity point of $T: A \rightarrow B$, which is also a fixed point if $A$ and $B$ intersect, is an approximate best proximity point of $T: A \rightarrow B$ while the converse is not true, in general. If we have two mappings $g: A \rightarrow A$ and $T: A \rightarrow B$ then:

(5) $x \in A$ is an optimal best proximity coincidence point of the pair $(g, T)$ if $d(g x, T x)=d(A, B)$;

(6) $x \in A$ is an approximate optimal best proximity coincidence point of the pair $(g, T)$ if $d(g x, T x)=$ $\inf \{d(g y, T x): y \in A\}$.

Note that optimal best proximity coincidence points are also approximate optimal best proximity coincidence points but the converse is not true, in general. Note also that, if $A$ and $B$ intersect, then an optimal best proximity coincidence point of the pair $(g, T)$ is also a coincidence point of $(g, T)$. The above concepts can be extended to the "fuzzy" framework formalism when dealing with fuzzy metric spaces. The purpose of this paper is to investigate some relevant properties of two types of fuzzy ordered proximal contractions of cyclic self-mappings $T$ integrated in a pair $(g, T)$ of mappings, where $g$ is a non-contractive fuzzy self-mapping and $T$ is a cyclic proximal contraction, in the framework of non-Archimedean ordered fuzzy complete metric spaces. In particular, the existence, uniqueness and limit properties for sequences of optimal fuzzy best proximity coincidence points are investigated for such pairs of mappings.

\section{Notation}

$\boldsymbol{R}$ is the set of real numbers, $\boldsymbol{R}_{+}=\{z \in \boldsymbol{R}: z>0\}, \boldsymbol{R}_{0+}=\boldsymbol{R}_{+} \cup\{0\}$;

$\boldsymbol{Z}$ is the set of integer numbers, $\boldsymbol{Z}_{+}=\{z \in \boldsymbol{Z}: z>0\}, \boldsymbol{Z}_{0+}=\boldsymbol{Z}_{+} \cup\{0\}$;

$\bar{p}=\{1, \cdots, p\}$;

$\mathrm{cl}($.$) is the closure of the (.)-set.$

The subsequent equality holds for the t-norm $*:[0,1]^{2} \rightarrow[0,1]$ for a fuzzy set $M$ on $X \times$ $X \times[0, \infty):$

$$
\stackrel{n}{\stackrel{*}{*}} M\left(x, y, t_{n}\right)=M\left(x, y_{1}, t_{m}\right) * M\left(z_{0}, z_{1}, t_{m+1}\right) * \ldots * M\left(z_{0}, z_{1}, t_{n}\right)
$$

Some useful technical definitions to be used are given below:

Definition 1 [1]. A binary operation $*:[0,1]^{2} \rightarrow[0,1]$ is said to be a continuous $t$-norm if:

(i) * is continuous, commutative and associative; 
(ii) $a * 1=1$ for all $a \in[0,1]$;

(iii) $a * b \leq c * d$ if $a \leq c$ and $b \leq d$.

The formalism of fuzzy sets was proposed by Zadeh [4]. The following formal definition of fuzzy sets on non-Archimedean fuzzy metric spaces will be used throughout this manuscript.

Definition $2[2,3]$. Let $X$ be a non-empty set and $*$ be a continuous $t$ - norm. A fuzzy set $M$ on $X \times X \times[0, \infty)$ is said to be a fuzzy metric on the non-Archimedean fuzzy metric space $(X, M, *)$ if for any $x, y, z \in X$, the following conditions hold:

(i) (i) $M(x, y, t)>0$;

(ii) $x=y$ if and only if $M(x, y, t)=1 ; \forall t \in \mathbf{R}_{+}$;

(iii) $M(x, y, t)=M(y, x, t)$;

(iv) $M(x, y, \max (t, s))=M(x, z, t) * M(z, y, s) ; \forall t, s \in \boldsymbol{R}_{+}$;

(v) $M(x, y,):.[0, \infty) \rightarrow[0,1]$ is left-continuous.

If the condition (iv) of Definition 2 is replaced with $M(x, y, t+s)=M(x, z, t) * M(z, y, s)$; $\forall t, s \in \boldsymbol{R}_{+}$then $(X, M, *)$ is a (Archimedean) fuzzy metric space and $M(x, y,$.$) is non-decreasing on$ $[0, \infty)$ and continuous on $X^{2} \times(0, \infty)$ [5]. If $t=s$ then $M(x, y, t)=M(x, z, t) * M(z, y, t)$; $\forall t \in \boldsymbol{R}_{+}$and $M$ is said to be the strong metric on $X$. Each fuzzy metric $M$ on $X$ generates a Hausdorff topology $\tau_{M}$ whose base is the family of open balls of members $B_{M}(x, \varepsilon, t)=$ $\{y \in X: M(x, y, t)>1-\varepsilon\}$ for $\varepsilon \in(0,1), t \in \boldsymbol{R}_{+}$, and a sequence $\left\{x_{n}\right\}$ converges to $x \in X$ with respect to $\tau_{M}$ if and only if $\lim _{n \rightarrow \infty} M\left(x_{n}, x, t\right)=1 ; \forall t \in \boldsymbol{R}_{+}$. Note that, since (iv) implies the above condition, any non-Archimedean fuzzy metric space is a fuzzy metric space.

Definition 3 [13]. Let $A$ and $B$ be two non-empty subsets of a non-Archimedean fuzzy metric space $(X, M, *)$. Define the sets $A_{0}(t)$ and $B_{0}(t)$ as:

$$
\begin{aligned}
& A_{0}(t)=\{x \in A: M(x, y, t)=M(A, B, t) \text { for some } y \in B\}, \\
& B_{0}(t)=\{y \in B: M(x, y, t)=M(A, B, t) \text { for some } x \in A\} .
\end{aligned}
$$

Definition 4 [39]. Let $\Psi$ be the set of all mappings $\psi:[0,1] \rightarrow[0,1]$ satisfying the following properties:

(i) $\psi(0)=0, \psi(1)=1$ and $\psi(t)>t$ for $t \in(0,1)$ and it is continuous in $(0,1)$,

(ii) $\lim _{n \rightarrow \infty} \psi^{n}(t)=1$ if and only if $t=1$.

A point $x$ in an abstract non-empty set $X$ will be said to be an optimal fuzzy best proximity coincidence point of the pair of mappings $(g, T)$, where $g: A \rightarrow A$ and $T: A \rightarrow B$, where $A$ and $B$ are non-empty subsets of $X$, if $M(g x, T x, t)=M(A, B, t)$, where $X$ is a non-empty set and $M$ is a fuzzy metric. The main paper body consists of other two sections. In such sections, some proximal contractions associated with pair $(g, T)$ where $g$ is a non-contractive self-mapping and $T$ is a $p$-cyclic fuzzy ordered proximal contractive self-mapping are formalized and some of their propertied, like convergence of sequences and existence and uniqueness of optimal fuzzy best proximity coincidence points are investigated. The obtained results and their discussion are split into two sections as follows. Section 2 introduces some necessary concepts in the fuzzy framework while some results are obtained and proved concerning the so-called optimal fuzzy best proximity coincidence points in partially ordered non-Archimedean fuzzy metric spaces for cyclic fuzzy order preserving proximal $\psi$-contractions of type I. Section 3 reformulates the above results for another type of proximal contractions, so-called type II. Both sections contain and discuss some illustrative examples. 


\section{Results and Discussion on Optimal Fuzzy Best Proximity Coincidence Points in Non-Archimedean Fuzzy Metric Spaces for Cyclic Fuzzy Order Preserving Proximal $\psi$-Contractions of Type I}

This section is devoted to give a framework related to the existence of best proximity coincidence points in partially ordered non- Archimedean fuzzy metric spaces for cyclic fuzzy order preserving proximal $\psi$-contractions of type I. A set of necessary definitions are given to set and prove the results and a set of "ad hoc" discussed examples is also given.

Definition 5. Let $A$ be a non-empty subset of a non-Archimedean fuzzy metric space $(X, M, *)$. A self-mapping $f$ on $A$ is said to be:

(1) a fuzzy isometry if $M(f x, f y, t)=M(x, y, t)$ for all $x, y \in A$ and $t \in \boldsymbol{R}_{+}$;

(2) fuzzy non-contractive if for any $x, y \in A$ and $t \in \boldsymbol{R}_{+}$, we have $M(f x, f y, t) \leq M(x, y, t)$.

Definition 6. Let $(X, \preceq)$ be a preordered set and let $X_{i} \subseteq X$ be non-empty sets; $\forall i \in \bar{p} . A p(\geq 2)$-cyclic mapping $T: \cup_{i \in \bar{p}} X_{i} \rightarrow \cup_{i \in \bar{p}} X_{i}$ is said to be non-decreasing, or order preserving with respect to a preorder relation $\preceq$ on $\cup_{i \in \bar{p}} X_{0 i}(t)$, if:

(a) the binary preorder relation $\preceq$ on $\cup_{i \in \bar{p}} X_{i}$ is a partial order relation on each set $X_{0 i}(t) ; \forall i \in \bar{p}$;

(b) for any $x, y$ in $X_{i}$ and any $i \in \bar{p}$ if $x \preceq y$ then $T x \preceq T y$.

Remark 1. Note that Definition 6 could be restated under stronger conditions with the binary preorder relation $\preceq$ holding on $\cup_{i \in \bar{p}} X_{i}$ while being a partial order relation on each set $X_{i} ; \forall i \in \bar{p}$. Note that $X_{i} \supseteq A_{i}(t)$; $\forall i \in \bar{p}$ and $\cup_{i \in \bar{p}} X_{i} \supseteq\left(X_{i} \cup_{j(\neq i) \in \bar{p}} X_{0 j}(t)\right)$. In this context, Definition 6 can be applied to the partially order preserving non-Archimedean fuzzy metric space $(X, M, *, \preceq)$ (i.e., $(X, \preceq)$ is a partially order preserving set and $(X, M, *)$ is a non-Archimedean fuzzy metric space, [38]) to a p-cyclic mapping $T: \cup_{i \in \bar{p}} X_{i} \rightarrow \cup_{i \in \bar{p}} X_{i}$ where $X_{i} \subseteq$ Xare non-empty sets; $\forall i \in \bar{p}$.

The concepts of order preserving, order reversing and monotone mappings $T: A \rightarrow B$ have been discussed in [11], where related results have been obtained. An "ad hoc" adaptation of the concept of order preserving for cyclic mappings is proposed in the subsequent definitions:

Definition 7. A $p(\geq 2)$-cyclic mapping $T: \cup_{i \in \bar{p}} X_{i} \rightarrow \cup_{i \in \bar{p}} X_{i}$ is said to be a (strong) p-cyclic fuzzy ordered proximal $\psi$-contraction of type I if for any $u, v \in X_{0 i}(t), x, y \in X_{i}$ and any given $i \in \bar{p}$, the following condition holds:

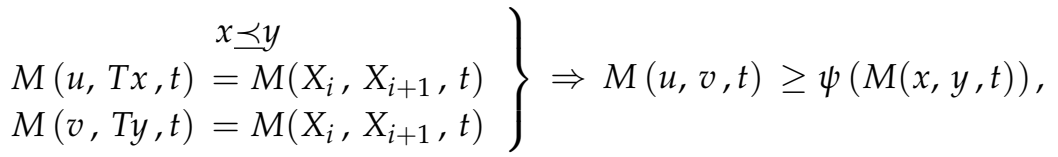

where $\psi \in \Psi$ for all $t \in \boldsymbol{R}_{+}$.

If the above conditions hold for any $u, v, x, y \in X_{0 i}(t)$ and any given $i \in \bar{p}$ then $T: \cup_{i \in \bar{p}} X_{i} \rightarrow \cup_{i \in \bar{p}} X_{i}$ is said to be a weak $p$-cyclic fuzzy ordered proximal $\psi$-contraction of type I.

Definition 8. $A p(\geq 2)$-cyclic mapping $T: \cup_{i \in \bar{p}} X_{i} \rightarrow \cup_{i \in \bar{p}} X_{i}$ is said to be a (strong) p-cyclic fuzzy ordered proximal $\psi$-contraction of type II if for some $\alpha \in(0,1)$, any $u, v \in X_{0 i}(t), x, y \in X_{i}$ and any given $i \in \bar{p}$, the following condition holds:

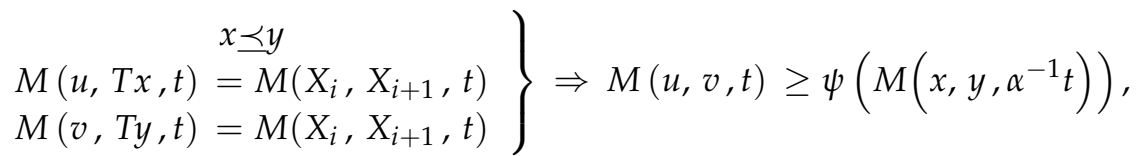

where $\psi \in \Psi$ for all $t \in \boldsymbol{R}_{+}$. 
If the above conditions hold for any $u, v, x, y \in X_{0 i}(t)$ and any given $i \in \bar{p}$ then $T: \cup_{i \in \bar{p}} X_{i} \rightarrow \cup_{i \in \bar{p}} X_{i}$ is said to be a weak $p$-cyclic fuzzy ordered proximal $\psi$-contraction of type II.

Definition 9. $A p(\geq 2)$-cyclic mapping $T: \cup_{i \in \bar{p}} X_{i} \rightarrow \cup_{i \in \bar{p}} X_{i}$ is said to be (strong) $p$-cyclic proximal fuzzy order preserving if for any $u, v \in X_{i}(t), x, y, z \in X_{i}$ and any given $i \in \bar{p}$ then $z \preceq T z$ and the following condition holds:

$$
\left.\begin{array}{c}
x \preceq y \\
M(u, T x, t)=M\left(X_{i}, X_{i+1}, t\right) \\
M(v, T y, t)=M\left(X_{i}, X_{i+1}, t\right)
\end{array}\right\} \Rightarrow u \preceq v ; \forall i \in \bar{p}
$$

If the above conditions hold for any $u, v, x, y, z \in X_{0 i}(t)$ and any given $i \in \bar{p}$ then $T: \cup_{i \in \bar{p}} X_{i} \rightarrow \cup_{i \in \bar{p}} X_{i}$ is said to be a weak $p$-cyclic proximal fuzzy order preserving mapping.

Definition 10 [20]. A point $z$ in an abstract non-empty set $X$ is said to be an optimal fuzzy best proximity coincidence point of the pair of mappings $(g, T)$, where $g: A \rightarrow A$ is a self-mapping and $T: A \rightarrow B$ is, in general, a non-self mapping, $A$ and $B$ are non-empty subsets of $X$ if $M(g z, T z, t)=M(A, B, t)$.

\section{Remark 2.}

(1) Note that Definition 10 is applicable to the case when the mapping $T: \cup_{i \in \bar{p}} X_{i} \rightarrow \cup_{i \in \bar{p}} X_{i}$ is $p$-cyclic (so that $T\left(X_{i}\right) \subseteq X_{i+1} ; \forall i \in \bar{p}$ ) and $g: \cup_{j \in \bar{p}} X_{j}\left|X_{i} \rightarrow g: \cup_{j \in \bar{p}} X_{j}\right| X_{i} ; \forall i \in \bar{p}$;

(2) Strong proximal contractions might be simply referred to as proximal contractions when no confusion is expected. Note from Definitions 8-10 that proximal contractions of types I and II are also weak proximal contractions of types I and II, respectively.

A quadruple $(X, M, *, \preceq)$ is called a partially ordered non-Archimedean fuzzy metric space if $(X, \preceq)$ is a partially ordered set and $(X, M, *)$ is a non-Archimedean fuzzy metric space. The following main result of this section holds:

Theorem 1. Let $(X, M, *, \preceq)$ be a complete partially ordered non-Archimedean fuzzy metric space and let $X_{i} \subseteq X$ be non-empty sets; $\forall i \in \bar{p}$ with $\preceq$ being a partial order defined on $\cup_{i \in \bar{p}} X_{0 i}(t)$. Let a $p(\geq 2)$ -cyclic mapping $T: \cup_{i \in \bar{p}} X_{i} \rightarrow \cup_{i \in \bar{p}} X_{i}$ be continuous and weak $p$-cyclic fuzzy order preserving (with respect to $\preceq$ ) proximal $\psi$-contraction of type I and let $g: \cup_{i \in \bar{p}} X_{i} \rightarrow \cup_{i \in \bar{p}} X_{i}$ be surjective, fuzzy non-contractive and inverse monotone mapping such that, for any $x, y \in \cup_{i \in \bar{p}} X_{0 i}(t), g x$ and $g y$ are comparable with respect to $\preceq$ only if $x, y$ are comparable. Suppose also that each pair of elements of $\cup_{i \in \bar{p}} X_{0 i}(t)$ has a lower bound and an upper bound and that for any $t>0, X_{0 i}(t)$ is non-empty, $T\left(X_{0 i}(t)\right) \subseteq X_{0, i+1}(t)$ and $X_{0 i}(t) \subseteq g\left(X_{0 i}(t)\right)$; $\forall i \in \bar{p}$. If for each given $x_{0 i} \in X_{0 i}(t)$ for each $i \in \bar{p}$, there exists some element $x_{1 i}$ in $X_{0 i}(t)$ such that:

$$
M\left(g x_{1 i}, T x_{0 i}, t\right)=M\left(X_{i}, X_{i+1}, t\right) \text { with } x_{0 i} \preceq x_{1 i}
$$

then there exists a unique element $x_{i}^{*} \in c l X_{0 i}(t)$ for each $i \in \bar{p}$ that is an optimal fuzzy best proximity coincidence point of the pair $(g, T)$ in $X_{i}$ such that $x_{i+1}^{*}=T x_{i}^{*} ; \forall i \in \bar{p}$, and then:

$$
M\left(g x_{i}^{*} x_{i+1}^{*}, t\right)=M\left(X_{i}, X_{i+1}, t\right) ; \forall i \in \bar{p}
$$

Furthermore, each of the subsequences $\left\{z_{i n}\right\} \subset\left\{z_{n}\right\} \cap X_{0 i}(t)$ for each $i \in \bar{p}$ for $n \in \mathbf{Z}_{+}$, with $z_{i 0}=z_{0} \in X_{0 i}(t)$, being defined by any given first element $z_{0} \in \cup_{i \in \bar{p}} X_{0 i}(t)$ so that the proximal constraint:

$$
M\left(g z_{n+1}, T z_{n}, t\right)=M\left(X_{j}, X_{j+1}, t\right)
$$


holds for any given $n \in Z_{0+}, \forall j \in \bar{p}$, is a Cauchy sequence that is convergent to the optimal fuzzy best proximity coincidence point $x_{i}^{*} \in c l X_{0 i}(t)$ (the closure of $\left.X_{0 i}(t)\right)$ of the pair $(g, T)$ in $X_{i}$ provided that the two subsequent conditions hold:

(1) $n_{j-1, k}+1 \leq n \leq n_{j k}$, with $n_{j k}+1-n_{j-1, k} \geq 2$, for $j=i, i+1, \ldots, i+p-2$, and $n_{i+p-2, k}+1 \leq$ $n \leq n_{i+p-1, k+1}$, with $n_{i+p-1, k+1}-n_{i+p-2, k} \geq 2 ; \forall i, j \in \bar{p}, \forall k \in Z_{0+}$;

(2) the chosen arbitrary $i \in \bar{p}$ is such that the initial points $\left(z_{0}, z_{1}\right) \in X_{0 i}(t) \times X_{0 i}(t)$ and that the sets of positive integers $\left\{n_{j k}-n_{j, k+1}\right\}$ for $j \in \bar{p}$ and $k \in Z_{0+}$ have $p$ positive upper-bounding integers $\overline{n_{j}} \geq n_{j k}-n_{j, k+1} ; \forall k \in Z_{0+}, \forall j \in \bar{p}$ with $n_{i-1,0}=-1$ and $n_{i 0} \geq 1$.

Proof. Denote the restricted mappings $T: \cup_{j \in \bar{p}} X_{j}\left|X_{i} \rightarrow \cup_{j \in \bar{p}} X_{j}\right| X_{i+1}$ and $g: \cup_{j \in \bar{p}} X_{j}\left|X_{i} \rightarrow \cup_{j \in \bar{p}} X_{j}\right| X_{i}$; $\forall i \in \bar{p}$ of the functions $T: \cup_{i \in \bar{p}} X_{i} \rightarrow \cup_{i \in \bar{p}} X_{i}$ and $g: \cup_{i \in \bar{p}} X_{i} \rightarrow \cup_{i \in \bar{p}} X_{i}$, respectively, by $T_{i}: X_{i} \rightarrow X_{i+1}$ and $g_{i}: X_{i} \rightarrow X_{i} ; \forall i \in \bar{p}$. Let $z_{0}=x_{0 i}$ arbitrary in $X_{0 i}(t)$ for the given $i \in \bar{p}$ and some $z_{1}=x_{1 i} \in X_{0 i}(t)$ for any given arbitrary $i \in \bar{p}$ be such that $z_{0} \preceq z_{1}$ and $M\left(g z_{1}, T z_{0}, t\right)=$ $M\left(X_{i}, X_{i+1}, t\right)$. Since $T z_{1} \in T\left(X_{0 i}(t)\right) \subseteq X_{0, i+1}(t)$ and $X_{0 i}(t) \subseteq g\left(X_{0, i+1}(t)\right), z_{2} \in X_{0 i}(t)$ exists such that $M\left(g z_{2}, T z_{1}, t\right)=M\left(X_{i}, X_{i+1}, t\right)$. Since $T: \cup_{i \in \bar{p}} X_{i} \rightarrow \cup_{i \in \bar{p}} X_{i}$ is weak $p$-cyclic proximal fuzzy order preserving and $z_{0} \preceq z_{1}$ then $g z_{1} \preceq g z_{2}$ and, since $g: \cup_{i \in \bar{p}} X_{i} \rightarrow \cup_{i \in \bar{p}} X_{i}$ is surjective and $X_{0 i}(t) \subseteq g\left(X_{0, i+1}(t)\right)$ then it follows that $z_{1} \preceq z_{2}$. Assume that this is not the case and proceed by contradiction. Since $g z_{1}$ and $g z_{2}$ are comparable, which holds, by hypothesis, only if $z_{1}$ and $z_{2}$ are comparable, then $z_{1} \succ z_{2}$ since $z_{1} \preceq z_{2}$ is assumed false. However, then $g z_{1} \succ g z_{2}$, which contradicts $g z_{1} \preceq g z_{2}$. Then, $z_{1} \preceq z_{2}$ and, as a result, $z_{0} \preceq z_{1} \preceq z_{2}$. Proceeding in the same way, we can build a sequence $\left\{z_{n}\right\}$ with $z_{n} \in X_{0 i}(t)$ for $1 \leq n\left(\in Z_{0+}\right) \leq n_{i 0}$, where $1 \leq n_{i 0}\left(\in Z_{0+}\right) \leq \bar{n}_{i}-1$, such that $M\left(g z_{n+1}, T z_{n}, t\right)=M\left(X_{i}, X_{i+1}, t\right) ; z_{n} \preceq z_{n-1} ; \forall n\left(\in Z_{+}\right) \in \bar{n}_{i 0}$. Then, take $z_{n_{i 0}+1}=T z_{n_{i 0}} \in X_{0, i+1}(t)$

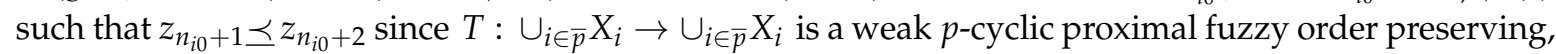
$z_{n_{i 0}} \in X_{0 i}(t)$ and $z_{n_{i 0}+1}=T z_{n_{i 0}} \in X_{0, i+1}(t)$. As a result, $z_{n-1} \preceq z_{n} ; \forall n\left(\in Z_{+}\right) \in \overline{n_{i 0}+1}$. Again,

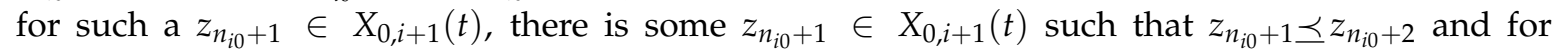
$1 \leq n_{i+1,0}\left(\in Z_{0+}\right) \leq \bar{n}_{i+1}$. Then, the elements of $\left\{z_{n}\right\}$ from $n=0$ to $n=n_{i 0}+2$ are ordered, with the order preserved with respect to the preorder relation $\preceq$ and $z_{n} \in X_{0 i}(t)$ and $z_{n_{i 0}+m} \in X_{0, i+1}(t)$ for $m=1,2$. By keeping $k=0$, we proceed in the same way by running $j$ from $i$ to $i+p-2$ and prove that the finite subsequence of $\left\{z_{n}\right\}$ from $n=0$ to $n=n_{i+p-2,0}+2$ is also totally ordered with respect to $\preceq$ since $n_{j-1,0}+1 \leq n \leq n_{j 0}$ for $j=i, i+1, \ldots, i+p-2 ; \forall j \in \bar{p}$ for the given $i \in \bar{p}$. Now, the same reasoning is used for $j=i+p-1$ and for $k=1$ and $n_{i+p-2,0}+1 \leq n \leq n_{i+p-1,1}$ to conclude that the elements of $\left\{z_{n}\right\}$ from $n=0$ to $n=n_{i+p-1,0}+2$ are ordered with respect to the relation $\preceq$. Proceeding recursively for each $j=i, i+1, \ldots, i+p-2$ and each integer $k \in Z_{+}$, it is proved that, $z_{n} \in X_{0 j}(t)$ for $n_{j-1, k}+1 \leq n \leq n_{j k}$ for $j=i, i+1, \ldots, i+p-2 ; \forall k \in Z_{0+}$ and $z_{n} \in X_{0 i}(t)\left(=X_{0, i+p-1}(t)\right)$ for $n_{i+p-2, k}+1 \leq n \leq n_{i+p-1, k+1} ; \forall k \in Z_{0+}$ and that the sequence $\left\{z_{n}\right\}$ is totally ordered with respect to $\preceq$. Define the strictly ordered set of positive integers $N^{*}=\left\{n_{j-1, k}\right\} ; j=i, i+1, \ldots, i+p-1$, $\forall k \in Z_{0+}$ with $n_{j k}<n_{j+1, k}$ and $n_{p, k}<n_{1, k+1} ; \forall j \in \bar{p}, \forall k \in Z_{0+}$. Since $T: \cup_{i \in \bar{p}} X_{i} \rightarrow \cup_{i \in \bar{p}} X_{i}$ is a weak $p$-cyclic proximal fuzzy order preserving (with respect to $\preceq$ ) proximal $\psi$-contraction of type I and $g: \cup_{i \in \bar{p}} X_{i} \rightarrow \cup_{i \in \bar{p}} X_{i}$ is fuzzy non-contractive, one has for $z_{n} \in\left\{z_{n}\right\}$ since $\psi:[0,1] \rightarrow[0,1]$ is in the set $\Psi$ :

$$
\tau_{n}(t)=M\left(z_{n}, z_{n+1}, t\right) \geq M\left(g z_{n}, g z_{n+1}, t\right) \geq \psi\left(M\left(z_{n-1}, z_{n}, t\right)\right)>\tau_{n-1}(t)=M\left(z_{n-1}, z_{n}, t\right)
$$

if $n,(n+1) \notin N^{*}$ and:

$$
\tau_{n}(t)=M\left(z_{n}, z_{n+1}, t\right) \geq M\left(g z_{n}, g z_{n+1}, t\right) \geq \psi\left(M\left(z_{n-1}, z_{n}, t\right)\right)>\tau_{n-1}(t)=M\left(z_{n-1}, z_{n}, t\right)
$$

if $(n+1),(n+2) \in N^{*}$. Note that if $z_{n-1}$ and $z_{n}$ are in $X_{0 j}(t)$ then $z_{n}$ and $z_{n+1}$ are in $X_{0 j}(t)$, for some $j \in \bar{p}$, if $n,(n+1) \notin N^{*}$ and in $X_{0, j+1}(t)(n+1),(n+2) \in N^{*}$ since, by hypothesis, $n_{j-1, k}+1 \leq$ 
$n \leq n_{j k}$, with $n_{j k}+1-n_{j-1, k} \geq 2$, for $j=i, i+1, \ldots, i+p-2$ and $n_{i+p-2, k}+1 \leq n \leq n_{i+p-1, k+1}$, with $n_{i+p-1, k+1}-n_{i+p-2, k} \geq 2 ; \forall i, j \in \bar{p}$. As a result, there is a subsequence $\left\{m_{j k}\right\}$ of non-negative integer numbers that depends on the initial $z_{0} \in X_{0 i}(t)$, such that $z_{m_{j k}}, z_{m_{j k}+1}$ and $z_{m_{j k}+1}$ are in $X_{0 j}(t) \cap\left\{z_{n}\right\}$ for all $k \in Z_{0+}$ and each $j \in \bar{p}$.

Also, for the subsequence $\left\{z_{m_{j k}+\ell}\right\} \subseteq\left\{z_{n}\right\}$ for $\ell=0,1,2 ; k \in Z_{0+}$ and any given $j \in \bar{p}$, $\left\{\tau_{m_{j n}+\ell}(t)\right\}$ and $\ell=0,1,2$ are strictly increasing sequences with $n \in Z_{0+}$ for each given $j \in \bar{p}$, so that $m_{j n}+\ell \rightarrow \infty$ as $n \rightarrow \infty$ for $\ell=0,1,2$, and convergent to a limit $\tau_{j}(t)$ in $(0,1]$ for each $t \in \boldsymbol{R}_{+}$and $j \in \bar{p}$ since $\psi:[0,1] \rightarrow[0,1]$ is continuous and non-decreasing and Equations (4) and (5) hold with:

$$
\begin{gathered}
1 \geq \tau_{m_{j n}+\ell}(t) \geq \psi\left(\tau_{m_{j, n-1}+\ell}(t)\right)>\tau_{m_{j, n-1}+\ell}(t) ; \forall n \in Z_{0+} \\
1 \geq \tau_{j}(t)=\lim _{n \rightarrow \infty} \tau_{m_{j n}+\ell}(t) \geq \lim _{n \rightarrow \infty} \tau_{m_{j n}+\ell}(t) ; \forall n \in Z_{0+}
\end{gathered}
$$

For each $j \in \bar{p}$. Assume that there is $t_{0} \in \boldsymbol{R}_{+}$such that $1>\tau_{j}\left(t_{0}\right)$ for some $j \in \bar{p}$. Then, the subsequent contradiction follows:

$$
\tau_{j}\left(t_{0}\right)=\lim _{n \rightarrow \infty} \tau_{m_{j n}+\ell}\left(t_{0}\right) \geq \psi\left(\tau_{j}\left(t_{0}\right)\right)>\tau_{j}\left(t_{0}\right)
$$

for each $j \in \bar{p}$. Thus, $\tau_{j}(t)=1$ for all $t \in \boldsymbol{R}_{+}$and all $j \in \bar{p}$. It is now proven that the subsequences $\left\{z_{m_{j n}}\right\} \subset X_{0 j}(t)$ are Cauchy sequences in $X_{0 j}(t)$ for each given $j \in \bar{p}$. Suppose that there is a sequence $\left\{z_{m_{j n}}\right\}$ that is not Cauchy for some $j \in \bar{p}$. Then, there exists $\varepsilon \in(0,1)$ and $t_{0} \in \boldsymbol{R}_{+}$such that for all $k \in Z_{0+}$, there are $n_{j n_{k}} m_{j n_{k}}\left(>n_{k}\right) \in \mathbf{Z}_{0+}$ such that $M\left(z_{n_{j n_{k}}}, z_{m_{j n_{k}}}, t_{0}\right) \leq 1-\varepsilon$; for some $j \in \bar{p}$. Assume that $m_{j n_{k}}$ is the least integer exceeding $n_{j n_{k}}$ and satisfying the above inequality so that:

$$
M\left(z_{m_{j, n_{k}-1}}, z_{n_{j n_{k}}}, t_{0}\right)>1-\varepsilon
$$

Then, one obtains for all $k \in Z_{0+}$ :

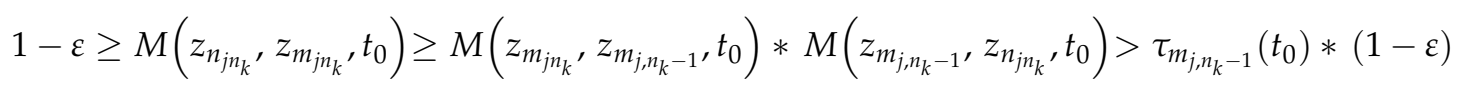

and, since $\lim _{k \rightarrow \infty} \tau_{m_{j, n_{k}-1}}\left(t_{0}\right)=\tau_{j}\left(t_{0}\right)=1$ for all $j \in \bar{p} *:[0,1]^{2} \rightarrow[0,1]$ is a continuous $t$-norm and $\varepsilon>0$, one gets by taking the limits in Equation (10) as $k \rightarrow \infty$ the following contradiction:

$$
1-\varepsilon>1 *(1-\varepsilon)=1-\varepsilon
$$

As a result, $\left\{z_{m_{j n}}\right\}$ is a Cauchy sequence for all $j \in \bar{p}$. Since $(X, M, *, \preceq)$ is complete, there exists $x_{j}^{*} \in c l X_{0 j}(t)$ such that $\left\{z_{m_{j n}}\right\} \rightarrow x_{j}^{*} ; \forall j \in \bar{p}$ since $m_{j n} \rightarrow \infty$ as $n \rightarrow \infty$ and $\lim _{n \rightarrow \infty} M\left(z_{m_{j n}}, x_{j}^{*}, t\right)=1$; $\forall j \in \bar{p}, \forall t \in \boldsymbol{R}_{+}$. Since $T: \cup_{i \in \bar{p}} X_{i} \rightarrow \cup_{i \in \bar{p}} X_{i}$ is continuous, this also implies that:

$$
\begin{gathered}
M\left(g x_{j}^{*}, T\left(T x_{j}^{*}\right), t\right)=\lim _{n \rightarrow \infty} M\left(T z_{m_{j n}}, g z_{m_{j, n+1}}, t\right)=\lim _{n \rightarrow \infty} M\left(T x_{j}^{*}, g z_{m_{j, n+1}}, t\right)=\lim _{n \rightarrow \infty} M\left(g x_{j}^{*}, T z_{m_{j n}}, t\right) \\
=M\left(X_{j}, X_{j+1}, t\right)
\end{gathered}
$$

so that $x_{j}^{*} \in c l X_{0 j}(t)$ is the common best proximity point of the pair $(g, T)$ in $X_{0 j}(t)$ for each $j \in \bar{p}$. It is now proven that $x_{j}^{*} \in c l X_{0 j}(t)$ is unique for each $j \in \bar{p}$. This is equivalent to proving that, for any fixed element $\bar{x}_{0}, x_{0} \in X_{0 j}(t)$, the subsequence $\left\{\bar{x}_{m_{j n}}\right\} \subset X_{0 j}(t)$ of $\left\{\bar{x}_{j n}\right\} \subset \cup_{j \in \bar{p}} X_{0 j}(t)$ converges to the same $x_{j}^{*} ; \forall j \in \bar{p}$. Since $T: \cup_{i \in \bar{p}} X_{i} \rightarrow \cup_{i \in \bar{p}} X_{i}$ is a weak $p$-cyclic fuzzy order preserving proximal $\psi$-contraction of type I and $g: \cup_{i \in \bar{p}} X_{i} \rightarrow \cup_{i \in \bar{p}} X_{i}$ is fuzzy non-contractive, one gets the subsequent contradiction under the assumption that $x_{j}^{*} \neq \bar{x}_{j}^{*}$ for some $j \in \bar{p}$ such that, if 
$x_{0} \neq \bar{x}_{0}$ and $x_{j}^{*}$ and $\bar{x}_{j}^{*}$ are comparable, the convergent subsequences $\left\{x_{m_{j n}}\right\}\left(\subset X_{0 j}(t) \cap\left\{x_{n}\right\}\right) \rightarrow x_{j}^{*}$, $\left\{x_{\bar{m}_{j n}}\right\}\left(\subset X_{0 j}(t) \cap\left\{\bar{x}_{n}\right\}\right) \rightarrow \bar{x}_{j}^{*}\left(\neq x_{j}^{*}\right)$, such that the sequences of nonnegative integers $\left\{m_{j n}\right\}$ and $\left\{\bar{m}_{j n}\right\}$ with $m_{j n}, \bar{m}_{j n} \rightarrow \infty$ as $n \rightarrow \infty ; \forall j \in \bar{p}$, defined by:

$$
M\left(g \bar{x}_{\bar{m}_{j n}+1}, T \bar{x}_{\bar{m}_{j n}+1}, t\right)=M\left(g x_{m_{j n}+1}, T x_{m_{j n}}, t\right)=M\left(X_{j}, X_{j+1}, t\right) ; \forall n \in Z_{0+}
$$

satisfy the set of inequalities:

$$
\begin{gathered}
M\left(x_{j}^{*}, \bar{x}_{j}^{*}, t\right) \geq M\left(g x_{j}^{*}, g \bar{x}_{j}^{*}, t\right) \\
\geq M\left(g x_{j}^{*}, T x_{j}^{*}, t\right) * M\left(T \bar{x}_{j}^{*}, T x_{j}^{*}, t\right) * M\left(g \bar{x}_{j}^{*}, T \bar{x}_{j}^{*}, t\right) \\
\geq 1 *\left(1 * M\left(T \bar{x}_{j}^{*}, T x_{j}^{*}, t\right)\right) \\
=1 * M\left(T \bar{x}_{j}^{*}, T x_{j}^{*}, t\right) \\
=M\left(T \bar{x}_{j}^{*}, T x_{j}^{*}, t\right) \\
\geq \psi\left(M\left(\bar{x}_{j}^{*}, x_{j}^{*}, t\right)\right) \\
>M\left(\bar{x}_{j}^{*}, x_{j}^{*}, t\right)
\end{gathered}
$$

so that $x_{j}^{*}=\bar{x}_{j}^{*} ; \forall j \in \bar{p}$. Now, assume that the corresponding elements of the sequences $\left\{x_{n}\right\}$ and $\left\{\bar{x}_{n}\right\}$, with distinct initial values $x_{0}$ and $\bar{x}_{0}$, are not all pair-wise comparable. By the hypothesis of the mapping $T$ being a fuzzy order preserving proximal $\psi$-contraction of type I and the hypothesis of the mapping $g$ being inverse monotone, both sequences are lower-bounded and upper-bounded by sequences $\left\{u_{n}\right\}$ and $\left\{\bar{u}_{n}\right\}$, with the corresponding subsequences $\left\{u_{m_{j n}}\right\}$ and $\left\{\bar{u}_{\bar{m}_{j n}}\right\}$, respectively, in any of the sets $X_{0 j}(t)$, which are constructed from:

$$
M\left(g u_{m_{j n}+1}, T u_{m_{j n}}, t\right)=M\left(g \bar{u}_{\bar{m}_{j n}+1}, T \bar{u}_{\bar{m}_{j n}}, t\right)=M\left(X_{j}, X_{j+1}, t\right) ; \forall j \in \bar{p}
$$

provided that $m_{j n}, m_{j n}+1 \in\left\{m_{j n}\right\} \subset \mathbf{Z}_{0+}, \bar{m}_{j n}, \bar{m}_{j n}+1 \in\left\{\bar{m}_{j n}\right\} \subset \mathbf{Z}_{0+} ; \forall j \in \bar{p}$ and have order comparison properties of the form $u_{m_{j n}} \preceq x_{m_{j n}} \preceq \bar{u}_{\bar{m}_{j n}}$ and $u_{m_{j n}} \preceq \bar{x}_{\bar{m} j n} \preceq \bar{u}_{\bar{m}_{j n}} ; \forall n \in Z_{0+}, \forall j \in \bar{p}$ since the sequences $\left\{x_{m_{j n}}\right\}$ and $\left\{\bar{x}_{\bar{m}_{j n}}\right\} ; \forall j \in \bar{p}$, are totally ordered, and also both lower-bounded and upper-bounded by the pair-wise comparable sequences $\left\{u_{m_{j n}}\right\}$ and $\left\{\bar{u}_{\bar{m}_{j n}}\right\}$, respectively, since they are convergent. Then, $\lim _{n \rightarrow \infty} u_{m_{j n}}=\lim _{n \rightarrow \infty} \bar{u}_{\bar{m}_{j n}}=\lim _{n \rightarrow \infty} \bar{x}_{\bar{m}_{j n}}=\lim _{n \rightarrow \infty} x_{m_{j n}}=x_{j}^{*} ; \forall j \in \bar{p}$.

It is now proved that $x_{j+1}^{*}=T x_{j}^{*} ; \forall j \in \bar{p}$. Since $x_{j+1}^{*}, T x_{j}^{*} \in X_{0, j+1}(t)$, then:

$$
M\left(g x_{j}^{*}, T\left(T x_{j}^{*}\right), t\right)=\lim _{n \rightarrow \infty} M\left(T \hat{z}_{m_{j+1, n}} g x_{j+1}^{*}, t\right)=M\left(T x_{j+1}^{*}, g x_{j+1}^{*}, t\right)=M\left(X_{j+1}, X_{j+2}, t\right)
$$

where $\left\{\hat{z}_{m_{j+1, n}}\right\} \subseteq X_{0, j+1}(t)$ is a Cauchy sequence of initial value $T x_{j}^{*}$ that is convergent to some $z_{j+1}^{*} \in \operatorname{cl} X_{0, j+1}(t)$, while another subsequence in $X_{0 j}(t)$ of a sequence with initial consecutive values $x_{0} \in X_{i}, x_{1} \in X_{0 i}(t)$ converges to $x_{j+1}^{*}=z_{j+1}^{*} \in X_{0, j+1}(t)$. However, since $x_{j}^{*} \in X_{0 j}(t)$ is unique and $T x_{j}^{*} \in X_{0, j+1}(t)$ for all $j \in \bar{p}$, then:

$$
M\left(X_{j+1}, X_{j+2}, t\right)=M\left(T x_{j+1}^{*}, g x_{j+1}^{*}, t\right)=M\left(T z_{j+1}^{*}, g x_{j+1}^{*}, t\right) ; \forall j \in \bar{p}
$$

since $z_{j+1}^{*}$ is a unique limit of Cauchy sequences in $X_{0, j+1}(t)$ then $z_{j+1}^{*}=T x_{j}^{*} ; \forall j \in \bar{p}$. So the unique limit of all Cauchy subsequences in $X_{0, j+1}(t)$ is $x_{j+1}^{*}=z_{j+1}^{*}=T x_{j}^{*} ; \forall j \in \bar{p}$.

Remark 3. Theorem 1 guarantees the existence of Cauchy sequences that are constructed from the proximal constraints and their convergence to unique optimal fuzzy best proximity coincidence points of the pair $(g, T)$ in 
$X_{i}$, which are located at $c l X_{0 i}(t) ; \forall i \in \bar{p}$ provided that the proximal constraints run at least for two consecutive iterations at each $X_{0 i}(t)$ before each iteration to the next adjacent subset $X_{0, i+1}(t)$ (see conditions 1-2). In the subsequent result, the constraints for running at least two consecutive proximal iterations at each $X_{0 i}(t)$; $\forall i \in \bar{p}$ are removed. Only a proximal iteration at $X_{0 j}(t)$ is needed for some given $j \in \bar{p}$ at particular cycles of the $p$-cyclic map T. This operation guarantees the convergence of the corresponding subsequences in $c l X_{0 i}(t)$; $\forall i \in \bar{p}$ to unique optimal fuzzy best proximity coincidence points of the pair $(g, T)$ at each set $X_{0 i}(t) ; \forall i \in \bar{p}$.

Corollary 1. Assume that the hypothesis of Theorem 1 holds except that, for any given initial point $z_{0} \in \cup_{i \in \bar{p}} X_{i}$, the sequences $\left\{z_{n}\right\}$ are built so that:

(1) $z_{n+1}=T z_{n}$ for any $n \in Z_{+}$if $z_{n} \in X_{0 i}(t)$ for any $i(\neq j) \in \bar{p}$ and some fixed $j \in \bar{p}$ (i.e., the proximal subsequence from each subset $X_{j}$ of $X$ to each next adjacent subset is only computed eventually at the subset $X_{0 j}(t)$, while at the remaining subsets only the cyclic self-mapping $T$ is involved);

(2) the proximal constraint Equation (3), subject to its subsequent constraints, is replaced at the subset $X_{j}$ of $X$ by the subsequent one:

$$
M\left(g z_{n_{j, k+m_{j}}+1}, T^{n_{j, k+m_{j}}+1-n_{j k}} z_{n_{j k}}, t\right)=M\left(X_{j}, X_{j+1}, t\right) ; \forall k\left(\geq k^{*}\right) \in \mathbf{Z}_{0+}, i \in \bar{p}
$$

for some given $j \in \bar{p}$ and some subsequence $\left\{z_{n_{j k}}\right\} \subseteq\left\{z_{n}\right\} \cap X_{0 j}(t)$, for $n \in Z_{+}$with $z_{0} \in X_{i}$ for some $i \in \hat{p}$ satisfying $z_{n_{j, k+m_{j}}}=T^{n_{j, k+m_{j}(k)}-n_{j k}} z_{n_{j k}}$ with $z_{n_{j} k^{*}}, z_{n_{j} k^{*}+1} \in X_{0 j}(t)$, for some set of bounded positive integers $m_{j}(k) ; \forall k\left(\geq k^{*}\right) \in Z_{0+}$ for the given $j \in \bar{p}$ and some sequence of positive integers $\left\{n_{j k}\right\}$ being strictly increasing with $k\left(\geq k^{*}\right) \in Z_{0+}$ (i.e., the proximal subsequence at the subset $X_{0 j}(t)$ is not necessary computed at each $k$-th cycle on the whole cyclic disposal of the $p$ subsets $X_{0 i}(t) \subseteq X_{i} \subseteq X$ for all $i \in \bar{p}$ since $m_{j}(k)$ can exceed the value $p$ for some values $k\left(\geq k^{*}\right) \in Z_{0+}$.

Then, there exists a unique element $x_{i}^{*} \in \operatorname{clX} X_{0 i}(t)$ for each $i \in \bar{p}$, which is an optimal fuzzy best proximity coincidence point of the pair $(g, T)$ in $X_{i}$, such that $x_{i+1}^{*}=T x_{i}^{*} ; \forall i \in \bar{p}$, and then $M\left(g x_{i}^{*} x_{i+1}^{*}, t\right)=M\left(X_{i}, X_{i+1}, t\right) ; \forall i \in \bar{p}$. Furthermore, each of the subsequences $\left\{z_{i n}\right\} \subset\left\{z_{n}\right\} \cap X_{0 i}(t)$ for $i \in \bar{p}$ being defined by any given first element $z_{0} \in \cup_{i \in \bar{p}} X_{0 i}(t)$, so that the proximal constraint $M\left(g z_{n+1}, T z_{n}, t\right)=M\left(X_{j}, X_{j+1}, t\right) ; \forall n \in Z_{0+}$, is a Cauchy sequence which is convergent to the optimal fuzzy best proximity coincidence point $x_{i}^{*} \in c l X_{0 i}(t)$ of the pair $(g, T)$ in $X_{i}$.

Sketch of Proof. Note that the proximal constraint (16) may be rewritten as:

$$
M\left(g z_{n_{j, k+m_{j}(k)}+1}, T z_{n_{j, k+m_{j}(k)}}, t\right)=M\left(X_{j}, X_{j+1}, t\right)
$$

by defining $z_{n_{j, k+m_{j}(k)}}=T^{n_{j, k+m_{j}(k)}-n_{j k}} z_{n_{j k}}$. Thus, we can define a strictly increasing sequence of nonnegative integers $\left\{\ell_{n}\right\}$ satisfying:

$$
\ell_{0}=n_{j k^{*}} \geq 0, \ldots, \ell_{n}=n_{j k_{n}}+m_{j}\left(k_{n}\right) \geq 0
$$

such that the nonnegative integers $m_{j}\left(k_{n}\right) \geq k_{n-1}-k_{n}+m_{j}\left(k_{n-1}\right)$ for all $n \in Z_{+}$and $m_{j}\left(k_{0}\right) \geq 0$, $k_{0}=k^{*} \in Z_{0+}, n_{j k_{1}} \in Z_{0+}$ with $n_{j k_{1}} \geq 0$ such that $z_{n_{j k_{0}}} \in X_{0 j}(t)$. Then, the subsequence $\left\{z_{\ell_{n}}\right\} \subset X_{0 j}(t)$ and satisfies the proximal condition (16). Then, according to Theorem 1, such a subsequence is Cauchy and convergent to a unique $x_{j}^{*} \in c l X_{0 j}(t)$, which is a unique optimal fuzzy best proximity coincidence points of the pair $(g, T)$ in $X_{j}$. Since this sequence is convergent, all subsequences $\left\{T^{k} z_{\ell_{n}}\right\} \rightarrow x_{j+k}^{*}$; $j=i, i+1, \ldots, i+p-1$ and $x_{j+k}^{*}$ is the unique limit point and also the unique optimal fuzzy best proximity coincidence point of the pair $(g, T)$ in $X_{i+k}$. 
Theorem 1 and Corollary 1 also hold if $T: \cup_{i \in \bar{p}} X_{i} \rightarrow \cup_{i \in \bar{p}} X_{i}$ is a $p$-cyclic fuzzy order preserving (strong) proximal $\psi$-contraction of type I such that the convergence of the constructed subsequences in each $X_{i}$ for $i \in \bar{p}$ converge asymptotically to be proximal subsequences converging to a unique optimal fuzzy best proximity coincidence point of $(g, T)$ in each $X_{i}$ so that $M\left(z_{n-1}, z_{n}, t\right) \rightarrow M\left(X_{i}, X_{i+1}, t\right)$ as $n \rightarrow \infty$. In particular, Equation (1) is replaced with Equation (17) below. A related result is as follows:

Corollary 2. Theorem 1 and Corollary 1 also hold "mutatis-mutandis" if $T: \cup_{i \in \bar{p}} X_{i} \rightarrow \cup_{i \in \bar{p}} X_{i}$ is a continuous $p$-cyclic fuzzy order preserving (with respect to $\preceq$ defined on $\cup_{i \in \bar{p}} X_{i}$ ) proximal $\psi$-contraction of type I provided that for each given for each $i \in \bar{p}$ and some existing element $x_{1 i}$ in $X_{i}$ :

$$
M\left(g x_{1 i}, T x_{0 i}, t\right) \leq M\left(X_{i}, X_{i+1}, t\right) \text { with } x_{0 i} \preceq x_{1 i}
$$

Sketch of Proof. Now take some arbitrary $z_{0}=x_{0 i}$ in $X_{i}$ and any arbitrary given $i \in \bar{p}$ and some $z_{1}=x_{1 i} \in X_{i}$ for any given arbitrary $i \in \bar{p}$ such that (17) holds (note that equality in Equation (17) holds if and only if $x_{0 i}, x_{1 i} \in X_{0 i}(t)$ ). Since $T: \cup_{i \in \bar{p}} X_{i} \rightarrow \cup_{i \in \bar{p}} X_{i}$ is a $p$-cyclic fuzzy order preserving proximal $\psi$-contraction of type I and $g: \cup_{i \in \bar{p}} X_{i} \rightarrow \cup_{i \in \bar{p}} X_{i}$ is fuzzy non-contractive, one has for any built subsequence $z_{n} \in\left\{z_{n}\right\} \subset X_{i}$ of the whole iteration, since $\psi:[0,1] \rightarrow[0,1]$ is in the set $\Psi$, and following Equations (4), (5) and (17):

$$
M\left(X_{i}, X_{i+1}, t\right) \geq M\left(z_{n}, z_{n+1}, t\right) \geq M\left(g z_{n}, g z_{n+1}, t\right) \geq \psi\left(M\left(z_{n-1}, z_{n}, t\right)\right)>M\left(z_{n-1}, z_{n}, t\right)
$$

if $n,(n+1) \notin N^{*}$, and:

$$
M\left(z_{n+1}, z_{n+2}, t\right)=M\left(T z_{n}, T z_{n+1}, t\right) \geq \psi\left(M\left(z_{n}, z_{n+1}, t\right)\right)>M\left(z_{n-1}, z_{n}, t\right)
$$

if $(n+1),(n+2) \in N^{*}$. One can conclude from the steps of the proof of Theorem 1 and from the sketch of the proof of Corollary 1 that $M\left(z_{n-1}, z_{n}, t\right) \rightarrow M\left(X_{i}, X_{i+1}, t\right)$ as $n \rightarrow \infty$ so that the subsequence $z_{n}$ converges to a best proximity point in $c l\left(X_{0 i}(t)\right)$, which is a unique optimal fuzzy best proximity coincidence point of $(g, T)$ in $X_{i}$.

Example 1. Let $X \subset \boldsymbol{R}_{0+}^{2}$ be defined by $X=X_{1} \cup X_{2}$, where $X_{1}=\left\{(0,1+x): x \in \boldsymbol{R}_{0+}\right\}$ and $X_{2}=\left\{(1+x, 0): x \in R_{0+}\right\}$. Note that $X_{1} \cap X_{2}=\varnothing$. Consider the complete ordered fuzzy metric space $(X, M, *, \preceq)$ under $M(x, y, t)=\frac{t}{t+d(x, y)} ; \forall x, y \in X, \forall t \in \boldsymbol{R}_{+}$and $M\left(X_{1}, X_{2}, t\right)=\frac{t}{t+1}$, where $d(x, y)=\left|x_{1}-y_{1}\right|+\left|x_{2}-y_{2}\right|$ for any $x=\left(x_{1}, x_{2}\right), y=\left(y_{1}, y_{2}\right) \in X$, and " $\preceq$ " is a coordinate-wise ordering for all $x, y, z, w \in X_{i}$ for $i=1,2$ defined by:

(a) $\quad(x, y) \preceq(z, w)$ if and only if $x \leq z$; and

(b) $\quad(x, y) \preceq T(x, y)$ for any $(x, y) \in X$, i.e., if $(x, y) \in X_{i}, T(x, y) \in X_{j}$ for $i, j(\neq i)=1,2$.

The proximal subsets are $X_{01}(t)=\{(0,1)\}, X_{02}(t)=\{(1,0)\}$.

Define the continuous2-cyclic mapping $T_{\lambda}\left(X_{1}\right)=(1+\lambda x, 0)$ and $T_{\lambda}\left(X_{2}\right)=(0,1+\lambda x)$ for some given real constant $\lambda \in[0,1)$. Note that $T_{\lambda}\left(X_{01}(t)\right)=X_{02}(t)$ and $T_{\lambda}\left(X_{02}(t)\right)=X_{01}(t) ; \forall \lambda \in$ $\boldsymbol{R}_{0+}$. If $\lambda=0$ then $T_{0}\left(X_{01}(t)\right)=X_{02}(t)=\{(1,0)\}$ and $T_{0}\left(X_{02}(t)\right)=X_{01}(t)=\{(0,1)\}$.

Define $g: X \rightarrow X$ as $g_{\rho}(0, x)=\rho(0, x)$ and $g_{\rho}(1+x, 0)=(1+\rho x, 0)$ for any given $x \in \boldsymbol{R}_{0+}$ and some given real $\rho \geq 1$. Note that $g_{\rho}\left(X_{01}(t)\right)=X_{01}(t), g_{\rho}\left(X_{02}(t)\right)=X_{02}(t)$ and that $g_{\rho}: X \rightarrow X$ is surjective and inverse monotone.

Now, we build a sequence $\left\{x_{n}\right\} \subset X_{1} \cup X_{2}$ by taking an initial point $x_{0}=\left(0, y_{0}\right) \in X_{1}$ for some real $y_{0}=1+x \geq 1$ for some given fixed real $x \in R_{0+}$. Now, $T_{\lambda} x_{0}=T_{\lambda}\left(0, y_{0}\right)=(1+\lambda x, 0)=$ $\left(1+\lambda\left(y_{0}-1\right), 0\right)$ for some given real $\lambda \in[0,1)$. Take $\psi(t)=\sqrt{t}$. Next, take some $y_{1} \in \boldsymbol{R}_{+}$such that $x_{1}=\left(0, y_{1}\right) \in X_{01}(t)$ and $x_{1} \preceq x_{0}$ (i.e., $\left.y_{1} \leq y_{0}\right), g_{\rho}\left(x_{1}\right)=\rho x_{1}=\rho\left(0, y_{1}\right)$ for some given real $\rho \in[1, \infty)$. 
In general, $x_{0} \in X_{1}, T x_{0} \in X_{2},\left\{x_{n}\right\} \subset X_{1},\left\{T_{\lambda} x_{n}\right\} \subset X_{2}$, with $T_{\lambda} x_{n}=\left(1+\lambda\left(y_{n}-1\right), 0\right)$, so that $x_{n+1} \preceq x_{n}$ for $n \in \mathbf{Z}_{+}$and:

$$
\begin{gathered}
M\left(g_{\rho} x_{n+1}, T_{\lambda} x_{n}, t\right)=M\left(\rho\left(0, y_{n+1}\right), T_{\lambda}\left(0, y_{n}\right), t\right) \\
=M\left(\left(0, \rho y_{n+1}\right),\left(1+\lambda\left(y_{n}-1\right), 0\right), t\right) \\
=\frac{t}{t+\left|\rho y_{n+1}+\lambda\left(1-y_{n}\right)-1\right|}
\end{gathered}
$$

Note that if $\rho=2$ and $\lambda \in[0,1)$ then $(\lambda / \rho) \in[0,1 / 2)$ and $\left\{y_{n}\right\}$ is strictly decreasing and $\left\{y_{n}\right\} \rightarrow 1$. Thus, for all $n \in \boldsymbol{Z}_{+}$, one has $M\left(g_{\rho} x_{n+1}, T_{\lambda} x_{n}, t\right) \geq M\left(g_{\rho} x_{n}, T_{\lambda} x_{n-1}, t\right)$, which converges to $M\left(X_{1}, X_{2}, t\right)=\frac{t}{t+1}$ as $n \rightarrow \infty, \forall t \in \boldsymbol{R}_{+}$, and:

$$
\begin{gathered}
M\left(x_{n+2}, x_{n+1}, t\right) \\
\geq M\left(g_{\rho} x_{n+2}, g_{\rho} x_{n+1}, t\right) \\
=M\left(\left(0, \rho y_{n+2}\right),\left(0, \rho y_{n+1}\right), t\right) \\
=\left(\frac{t}{t+\rho\left|y_{n+2}-y_{n+1}\right|}\right) \\
\geq \psi\left(M\left(\left(0, y_{n+1}\right),\left(0, y_{n}\right), t\right)\right) \\
=\left(\frac{t}{t+\left|y_{n+1}-y_{n}\right|}\right){ }^{1 / 2} \\
\geq\left(\frac{t}{t+\left|y_{n+1}-y_{n}\right|}\right)
\end{gathered}
$$

$\forall n \in \boldsymbol{Z}_{0+}, \forall t \in \boldsymbol{R}_{+}$, the last inequality being strict if $y_{n+1} \neq y_{n}$, with $\psi(0)=0$ while $\psi(1)=1$ if and only if $y_{n+1}=y_{n}$. Note that the sequence $\left\{M\left(x_{n+1}, x_{n}, t\right)\right\}$ is strictly increasing for all $t \in \boldsymbol{R}_{+}$and $M\left(x_{n+2}, x_{n+1}, t\right) \rightarrow 1$ as $n \rightarrow \infty, \forall t \in \boldsymbol{R}_{+}$so that $\left\{y_{n+1}-y_{n}\right\} \rightarrow 0, x_{n+1} \preceq x_{n}, \forall n \in \mathbf{Z}_{0+}$, and:

$$
\left\{x_{n}\right\}\left(\subset X_{01}(t)\right) \rightarrow(0,1),\left\{T_{\lambda} x_{n}\right\}\left(\subset X_{02}(t)\right) \rightarrow(1,0)
$$

Note that this example extends the validity of Corollary 1-via Corollary 2-to the construction of sequences in the whole $X_{1}$ (instead of on just the proximal subset $X_{01}(t) \subset X_{1}$ ), which converge to the unique proximal point limit $(0,1) \in X_{01}(t)$ to $X_{2}$.

Example 2. If, in Example 1, we take the initial points either in the proximal set $X_{01}(t)=\{0,1\}$ or in the proximal set $X_{02}(t)=\{1,0\}$, i.e., $x_{0} \in\{\{0,1\}\{1,0\}\}$, then, according to Corollary $1, T_{\lambda}(0,1)=(1,0)$ and $T_{\lambda}(1,0)=(0,1)$ since $T_{\lambda}\left(X_{1}\right)=(1+\lambda x, 0)$ and $T_{\lambda}\left(X_{2}\right)=(0,1+\lambda x)$ for $x \geq 0$.

Example 3. Note that, in Example 1, we have taken the initial conditions in $X_{1}$ and the proximal sequences to $X_{2}$ are always constructed within $X_{1}$ and converge to the unique best proximity point of the proximal set $X_{01}(t)=\{0,1\} \subset X_{1}$ to $X_{2}$ so that the best proximity point in $X_{2}$ is focused as a limit point via the cyclic mapping since $T_{\lambda}\left(X_{01}(t)\right)=X_{02}(t)=\{1,0\} \subset X_{2}$. In Example 2, the points of the proximal built sequences are taken directly on both proximal sets. Now, we can construct converging proximal sequences with elements in both $X_{1}$ and $X_{2}$ that converge to the unique optimal fuzzy best proximity coincidence points of the pair $(g, T)$ in both corresponding proximal sets. Take a point $x_{0}=(1+\lambda x, 0) \in X_{2}$. We now proceed by constructing $a$ sequence with two consecutive elements in $X_{2}$, then the next one is in $X_{1}$ and again two consecutive proximal elements to $X_{2}$ in $X_{1}$ and so on so that the cyclic mapping $T$ is also relevant to alternate elements with two or more elements of the sequences of interest in both subsets in the cyclic disposal. Thus, for instance, the sequence:

$$
\begin{gathered}
\left\{(1+\lambda x, 0),\left(\frac{1+\lambda^{2} x}{\rho}, 0\right),\left(0, \frac{1+\lambda^{3} x}{\rho}\right),\left(0, \frac{1+\lambda^{4} x}{\rho^{2}}\right),\left(0, \frac{1+\lambda^{5} x}{\rho^{3}}\right),\right. \\
\left.\left(\frac{1+\lambda^{6} x}{\rho^{3}}, 0\right),\left(\frac{1+\lambda^{7} x}{\rho^{4}}, 0\right),\left(\frac{1+\lambda^{8} x}{\rho^{5}}, 0\right), \ldots,\right\}
\end{gathered}
$$

consists of two subsequences that converge to the best proximity points $(1,0)$ and $(0,1)$ for any given $x \in \boldsymbol{R}_{0+}$, which are the unique optimal fuzzy best proximity coincidence points of $(g, T)$. 


\section{Results and Discussion of Optimal Fuzzy Best Proximity Coincidence Points in Partially Ordered Non-Archimedean Fuzzy Metric Spaces for Cyclic Fuzzy Order Proximal $\psi$-Contractions of Type II}

This section is devoted to a framework related to the existence of best proximity coincidence points in partially ordered non-Archimedean fuzzy metric spaces for cyclic fuzzy order preserving proximal $\psi$-contractions of type II. Three definitions are given; we then state and prove the results and two examples are also given.

The following definitions are used in the main results of this section:

Definition 11 [8]. A sequence $\left\{t_{n}\right\}$ of positive real numbers is said to be s-increasing if there exists $n_{0} \in \mathbf{Z}_{0+}$ such that $t_{n+1}=t_{n}+1$ for all $n \geq n_{0}$.

Definition 12 [7,8]. A fuzzy metric space $(X, M, *)$ is said to have the property $T$ if for any s-increasing sequence $\left\{t_{n}\right\}$ and any given real constant $\varepsilon \in(0,1)$, there exists $n_{0}=n_{0}(\varepsilon) \in Z_{0+}$ such that

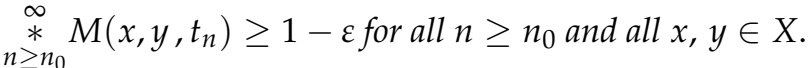

An alternative definition under weaker conditions follows:

Definition 13. A fuzzy metric space $(X, M, *)$ is said to have the property $T_{0}$ if for any $s$-increasing sequence $\left\{t_{n}\right\}$ and any given real constant $\varepsilon \in(0,1)$, there exist some $n_{0}=n_{0}(\varepsilon) \in Z_{0+}$ and some $N \in Z_{+}$such that:

$$
\underset{\substack{n_{0}+N-1 \\ n \geq n_{0}}}{*} M\left(x, y, t_{n}\right) \geq 1-\varepsilon \text { for all } n \geq n_{0} ; \forall x, y \in X
$$

The following result relates the properties $T_{0}$ and $T$ if $\lim _{t \rightarrow \infty} M(x, y, t)=1 ; \forall x, y \in X$.

Lemma 1. Let $(X, M, \bullet)$ be a fuzzy metric space endowed with the product $t$-norm $\bullet:[0,1]^{2} \rightarrow[0,1]$ such that its associate fuzzy metric fulfils $\lim _{t \rightarrow \infty} M(x, y, t)=1 ; \forall x, y \in X$. Then, the fuzzy metric space $(X, M, *)$, endowed with a $t$-norm $*:[0,1]^{2} \rightarrow[0,1]$ has the properties $T_{0}$ and $T$ for any $t$-norm $*:[0,1]^{2} \rightarrow[0,1]$.

Proof. If $\lim _{t \rightarrow \infty} M(x, y, t)=1$ for any $s$-increasing sequence $\left\{t_{n}\right\}$, one has:

$$
\lim _{n \rightarrow \infty} M\left(x, y, t_{n}\right)=\lim _{n \rightarrow \infty} M\left(x, y, t_{n+\ell}\right)=\lim _{n, m \rightarrow \infty} M\left(x, y, t_{n+m}\right)=1 ; \forall x, y \in X, \forall \ell \in Z_{0+}
$$

and for any given real constant $\varepsilon_{1} \in(0,1)$, there exists $n_{0}=n_{0}\left(\varepsilon_{1}, *\right) \in \mathbf{Z}_{0+}$ such that $M\left(x, y, t_{n}\right) \geq$ $1-\varepsilon_{1}$ for any $x, y \in X$ and $n \geq n_{01}$ since $M(x, y,$.$) is a non-decreasing function on (0, \infty)$. Also, for any given real constants $\varepsilon \in(0,1)$ and $\varepsilon_{1} \in\left(0, \bar{\varepsilon}_{1}\right)$, and some integer $N \in \boldsymbol{Z}_{+}$, where $\bar{\varepsilon}_{1}=\bar{\varepsilon}_{1}(\varepsilon, N)<$ $1-(1-\varepsilon)^{1 / N+1}$ is in $(0,1)$, one gets for the fuzzy metric space $(X, M, \bullet)$ :

$$
\underset{n \geq n_{0}}{n_{0}+N-1}\left[M\left(x, y, t_{n}\right)\right] \geq\left(1-\varepsilon_{1}\right)^{N} \geq 1-\varepsilon \text { for some } N=N\left(\varepsilon, \varepsilon_{1}\right) \in Z_{0+}
$$

and $\bar{\varepsilon}_{1}=\bar{\varepsilon}_{1}(\varepsilon, \infty) \in(0,1) ; \forall x, y \in X$ so that $(X, M, \bullet)$ possesses the property $T_{0}$. Since $\lim _{n \rightarrow \infty} M\left(x, y, t_{n}\right)=1 ; \forall x, y \in X$ then $\lim _{n \rightarrow \infty}{ }_{\substack{\bullet+N \\ m=n}}^{n+1} M\left(x, y, t_{m}\right)=\lim _{n, S \rightarrow \infty}{ }_{m=n}^{n+S-1} M\left(x, y, t_{m}\right)=1$; $\forall x, y \in X$. Thus, since $(X, M, \bullet)$ has the property $\boldsymbol{T}_{0}$ then for any s-increasing sequence $\left\{t_{n}\right\}$ and any 
given real constant $\varepsilon \in(0,1)$, there exists $n_{0}=n_{0}(\varepsilon) \in Z_{0+}$ such that $\underset{n \geq n_{0}}{n_{0}+N-1} M\left(x, y, t_{n}\right) \geq 1-\varepsilon$ for all integer $n \geq n_{0} ; \forall x, y \in X$. As a result, $(X, M, \bullet)$ has the property $T$ since:

$$
\stackrel{\stackrel{\bullet}{\bullet}}{m=n\left(\geq n_{0}\right)} M\left(x, y, t_{m}\right)=\lim _{N \rightarrow \infty}\left(\underset{m=n\left(\geq n_{0}\right)}{\stackrel{n+N-1}{\bullet}} M\left(x, y, t_{m}\right)\right) * \lim _{N \rightarrow \infty}\left({\underset{m=n+N}{n+2 N-1}}_{m=n}^{\bullet} M\left(x, y, t_{m}\right)\right) \geq(1-\varepsilon) \bullet 1=1-\varepsilon
$$

If the metric space is $(X, M, \Delta)$, with $\Delta:[0,1]^{2} \rightarrow[0,1]$ being the triangular $t$-norm, then one gets for any arbitrary $\varepsilon=\varepsilon_{1} \in(0,1)$ that $\underset{n \geq n_{0}}{\Delta}\left[M\left(x, y, t_{n}\right)\right] \geq 1-\varepsilon_{1}=1-\varepsilon$.

Since the triangular $t$-norm exceeds any other $t$-norm, all of which are strictly larger than the drastic $t$-norm $*_{D}:[0,1]^{2} \rightarrow[0,1]$, one gets from the above results for any given $\varepsilon \in(0,1)$ and $\varepsilon_{1} \in\left(0, \bar{\varepsilon}_{2}\right)$ with $\bar{\varepsilon}_{2}=\min \left(\bar{\varepsilon}_{1}, 1-\underset{\substack{n_{0}+N-1 \\ * D \\ n \geq n_{0}}}{*}\left[M\left(x, y, t_{n}\right)\right]\right)$, and some integer $N \in Z_{+}$, where $\bar{\varepsilon}_{1}=\bar{\varepsilon}_{1}(\varepsilon, N)<1-(1-\varepsilon)^{1 / N+1}$ is in $(0,1)$, that:

$$
\begin{aligned}
& \underset{n \geq n_{0}}{n_{0}+N-1}\left[M\left(x, y, t_{n}\right)\right] \geq \underset{\substack{n_{0}+N-1 \\
n \geq n_{0}}}{\stackrel{*}{*}}\left[M\left(x, y, t_{n}\right)\right] \geq \underset{n \geq n_{0}}{\stackrel{n_{0}+N-1}{\bullet}}\left[M\left(x, y, t_{n}\right)\right] \\
& \geq\left(1-\varepsilon_{1}\right)^{N} \geq 1-\varepsilon \geq \underset{\substack{n_{0}+N-1 \\
* D \\
n \geq n_{0}}}{*}\left[M\left(x, y, t_{n}\right)\right] \geq 0
\end{aligned}
$$

and $(X, M, *)$ has the property $T_{0}$ for any $t$-norm $*:[0,1]^{2} \rightarrow[0,1]$. Also, $\underset{n \geq n_{0}}{\stackrel{n_{0}+N-1}{*}} M\left(x, y, t_{n}\right) \geq$

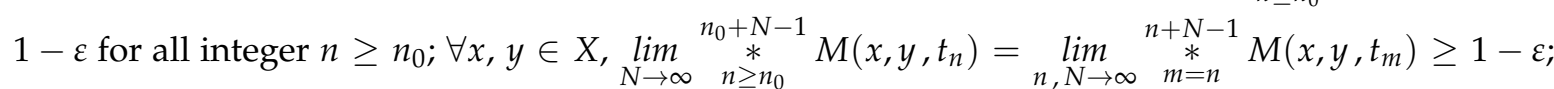
$\forall x, y \in X$ for any $t$-norm $*:[0,1]^{2} \rightarrow[0,1]$ so that $(X, M, *)$ has also the property $T$.

Lemma 1 concludes that property $T$ is less restrictive than property $T_{0}$ and holds under a standard property in probabilistic spaces $\lim _{t \rightarrow \infty} M(x, y, t)=1 ; \forall x, y \in X$. The subsequent result is close to Theorem 1 for the case when $T: \cup_{i \in \bar{p}} X_{i} \rightarrow \cup_{i \in \bar{p}} X_{i}$ is a weak $p$-cyclic fuzzy order preserving proximal $\psi$-contraction of type II. The main result of this section follows below:

Theorem 2. Let $(X, M, *, \preceq)$ be a complete partially ordered non-Archimedean fuzzy metric space satisfying the property $T$ and let $X_{i} \subseteq X$ be non-empty sets; $\forall i \in \bar{p}$ with $\preceq$ being a partial order defined on $\cup_{i \in \bar{p}} X_{0 i}(t)$. Let a $p(\geq 2)$-cyclic mapping $T: \cup_{i \in \bar{p}} X_{i} \rightarrow \cup_{i \in \bar{p}} X_{i}$ be continuous and weak $p$-cyclic fuzzy order preserving (with respect to $\preceq$ ) proximal $\psi$-contraction of type II and let $g: \cup_{i \in \bar{p}} X_{i} \rightarrow \cup_{i \in \bar{p}} X_{i}$ be surjective, fuzzy non-contractive and inverse monotone mapping such that, for any $x, y \in \cup_{i \in \bar{p}} X_{0 i}(t), g x$ and gy are comparable with respect to $\preceq$ only if $x, y$ are comparable. Suppose also that each pair of elements of $\cup_{i \in \bar{p}} X_{0 i}(t)$ has a lower bound and an upper bound, and that for any $t>0, X_{0 i}(t)$ is non-empty, $T\left(X_{0 i}(t)\right) \subseteq X_{0, i+1}(t)$ and $X_{0 i}(t) \subseteq g\left(X_{0 i}(t)\right) ; \forall i \in \bar{p}$. If for each given $x_{0 i} \in X_{0 i}(t)$ for each $i \in \bar{p}$, there exist some element $x_{1 i}$ in $c l X_{0 i}(t)$ such that:

$$
M\left(g x_{1 i}, T x_{0 i}, t\right)=M\left(X_{i}, X_{i+1}, t\right) \text { with } x_{0 i} \preceq x_{1 i}
$$

Then, there exists a unique element $x_{i}^{*} \in c l X_{0 i}(t)$ for each $i \in \bar{p}$, which is an optimal fuzzy best proximity coincidence point of the pair $(g, T)$ in $X_{i}$ such that $x_{i+1}^{*}=T x_{i}^{*} ; \forall i \in \bar{p}$, and then $M\left(g x x_{i}^{*} x_{i+1}^{*}, t\right)=M\left(X_{i}, X_{i+1}, t\right) ; \forall i \in \bar{p}$. Furthermore, each of the subsequences $\left\{z_{i n}\right\} \subset X_{0 i}(t)$ for each $i \in \bar{p}$ being defined by any given first element $z_{0} \in \cup_{i \in \bar{p}} X_{0 i}(t)$ so that the proximal constraint:

$$
M\left(g z_{n+1}, T z_{n}, t\right)=M\left(X_{j}, X_{j+1}, t\right)
$$


holds for any given $n \in Z_{0+}, \forall j \in \bar{p}$, is a Cauchy sequence which is convergent to the optimal fuzzy best proximity coincidence point $x_{i}^{*} \in X_{0 i}(t)$ of the pair $(g, T)$ in $X_{i}$ provided that the two subsequent conditions hold:

(1) $n_{j-1, k}+1 \leq n \leq n_{j k}$, with $n_{j k}+1-n_{j-1, k} \geq 2$, for $j=i, i+1, \ldots, i+p-2$, and $n_{i+p-2, k}+1 \leq$ $n \leq n_{i+p-1, k+1}$, with $n_{i+p-1, k+1}-n_{i+p-2, k} \geq 2 ; \forall i, j \in \bar{p}, \forall k \in Z_{0+}$;

(2) the chosen arbitrary $i \in \bar{p}$ is such that the initial point $z_{0} \in X_{0 i}(t)$ and that the sets of positive integers $\left\{n_{j k}-n_{j, k+1}\right\}$ for $j \in \bar{p}$ and $k \in Z_{0+}$ have $p$ positive upper-bounding integers $\overline{n_{j}} \geq n_{j k}-n_{j, k+1} ; \forall k \in Z_{0+}, \forall j \in \bar{p}$ with $n_{i-1,0}=-1$ and $n_{i 0} \geq 1$.

Proof. Denote the restricted mappings $T: \cup_{j \in \bar{p}} X_{j}\left|X_{i} \rightarrow \cup_{j \in \bar{p}} X_{j}\right| X_{i+1}$ and $g: \cup_{j \in \bar{p}} X_{j}\left|X_{i} \rightarrow \cup_{j \in \bar{p}} X_{j}\right| X_{i}$; $\forall i \in \bar{p}$ of the functions $T: \cup_{i \in \bar{p}} X_{i} \rightarrow \cup_{i \in \bar{p}} X_{i}$ and $g: \cup_{i \in \bar{p}} X_{i} \rightarrow \cup_{i \in \bar{p}} X_{i}$, respectively, by $T_{i}: X_{i} \rightarrow X_{i+1}$ and $g_{i}: X_{i} \rightarrow X_{i} ; \forall i \in \bar{p}$. Let $z_{0}=x_{0 i}$ arbitrary in $X_{0 i}(t)$ and some $z_{1}=x_{1 i} \in X_{0 i}$ for any given arbitrary $i \in \bar{p}$ be such that $z_{0} \preceq z_{1}$ and $M\left(g z_{1}, T z_{0}, t\right)=M\left(X_{i}, X_{i+1}, t\right)$. Since $T z_{1} \in T\left(X_{0 i}(t)\right) \subseteq X_{0, i+1}(t)$ and $X_{0 i}(t) \subseteq g\left(X_{0, i+1}(t)\right)$, one gets that $z_{2} \in X_{0 i}(t)$ exists such that $M\left(g z_{2}, T z_{1}, t\right)=M\left(X_{i}, X_{i+1}, t\right)$. Since $T: \cup_{i \in \bar{p}} X_{i} \rightarrow \cup_{i \in \bar{p}} X_{i}$ is a weak $p$-cyclic fuzzy order preserving proximal mapping and $z_{0} \preceq z_{1}$ then $g z_{1} \preceq g z_{2}$ and, since $g: \cup_{i \in \bar{p}} X_{i} \rightarrow \cup_{i \in \bar{p}} X_{i}$ is surjective and $X_{0 i}(t) \subseteq g\left(X_{0, i+1}(t)\right)$ then it follows that $z_{1} \preceq z_{2}$. Assume that this is not the case and proceed by contradiction. Since $g z_{1}$ and $g z_{2}$ are comparable which holds, by hypothesis, only if $z_{1}$ and $z_{2}$ are comparable then $z_{1} \succ z_{2}$ since $z_{1} \preceq z_{2}$ is assumed false. However, $g z_{1} \succ g z_{2}$ contradicts $g z_{1} \preceq g z_{2}$. Then, $z_{1} \preceq z_{2}$ and, as a result, $z_{0} \preceq z_{1} \preceq z_{2}$. Proceeding in the same way, we can build a sequence $\left\{z_{n}\right\}$ with $z_{n} \in X_{0 i}(t)$ for $0 \leq n\left(\in Z_{0+}\right) \leq n_{i 0}$, where $1 \leq n_{i 0}\left(\in Z_{0+}\right) \leq \bar{n}_{i}-1$, such that $M\left(g z_{n+1}, T z_{n}, t\right)=M\left(X_{i}, X_{i+1}, t\right) ; z_{n-1} \preceq z_{n} ; \forall n\left(\in Z_{+}\right) \in \bar{n}_{i 0}$. Then, take $z_{n_{i 0}+1}=T z_{n_{i 0}} \in X_{0, i+1}(t)$ such that $z_{n_{i 0}+1 \preceq} z_{n_{i 0}+2}$ since $T: \cup_{i \in \bar{p}} X_{i} \rightarrow \cup_{i \in \bar{p}} X_{i}$ is a weak $p$-cyclic fuzzy order preserving proximal mapping, $z_{n_{i 0}} \in X_{0 i}(t)$ and $z_{n_{i 0}+1}=T z_{n_{i 0}} \in X_{0, i+1}(t)$. As a result, $z_{n-1} \preceq z_{n} ; \forall n\left(\in Z_{+}\right) \in \overline{n_{i 0}+1}$. Again, for such a $z_{n_{i 0}+1} \in X_{0, i+1}(t)$, there is some $z_{n_{i 0}+1} \in X_{0, i+1}(t)$ such that $z_{n_{i 0}+1} \preceq z_{n_{i 0}+2}$ and for $1 \leq n_{i+1,0}\left(\in Z_{0+}\right) \leq \bar{n}_{i+1}$. Then, the elements of $\left\{z_{n}\right\}$ from $n=0$ to $n=n_{i 0}+2$ are ordered, with order preserving, with respect to the preorder relation $\preceq$ and $z_{n} \in X_{0 i}(t)$ and $z_{n_{i 0}+m} \in X_{0, i+1}(t)$ for $m=1,2$. By keeping $k=0$, we proceed in the same way by running $j$ from $i$ to $i+p-2$ and prove that the finite subsequence of $\left\{z_{n}\right\}$ from $n=0$ to $n=n_{i+p-2,0}+2$ is also ordered with respect to $\preceq$ since $n_{j-1,0}+1 \leq n \leq n_{j 0}$ for $j=i, i+1, \ldots, i+p-2 ; \forall j \in \bar{p}$ for the given $i \in \bar{p}$. Now, the same reasoning is used for $j=i+p-1$ and for $k=1$ and $n_{i+p-2,0}+1 \leq n \leq n_{i+p-1,1}$ to conclude that the elements of $\left\{z_{n}\right\}$ from $n=0$ to $n=n_{i+p-1,0}+2$ are ordered with respect to the relation $\preceq$. Proceeding recursively for each $j=i, i+1, \ldots, i+p-2$ and each integer $k \in Z_{+}$, it is proved that, $z_{n} \in X_{0 j}(t)$ for $n_{j-1, k}+1 \leq n \leq n_{j k}$ for $j=i, i+1, \ldots, i+p-2 ; \forall k \in Z_{0+}$ and $z_{n} \in X_{0 i}(t)\left(=X_{0, i+p-1}(t)\right)$ for $n_{i+p-2, k}+1 \leq n \leq n_{i+p-1, k+1} ; \forall k \in Z_{0+}$ and that the sequence $\left\{z_{n}\right\}$ is ordered with respect to $\preceq$. Define the strictly ordered set of positive integers $N^{*}=\left\{n_{j-1, k}\right\} ; j=i, i+1, \ldots, i+p-1$, $\forall k \in Z_{0+}$ with $n_{j k}<n_{j+1, k}$ and $n_{p, k}<n_{1, k+1} ; \forall j \in \bar{p}, \forall k \in Z_{0+}$. Since $T: \cup_{i \in \bar{p}} X_{i} \rightarrow \cup_{i \in \bar{p}} X_{i}$ is a weak $p$-cyclic fuzzy order preserving (with respect to $\preceq$ ) proximal $\psi$-contraction of type II and $g: \cup_{i \in \bar{p}} X_{i} \rightarrow \cup_{i \in \bar{p}} X_{i}$ is fuzzy non-contractive, one has for $z_{n} \in\left\{z_{n}\right\}$ and some real constant $\alpha \in(0,1)$, since $\psi:[0,1] \rightarrow[0,1]$ belongs to the set $\Psi$ :

$$
\begin{gathered}
M\left(z_{n}, z_{n+1}, t\right) \geq M\left(g z_{n}, g z_{n+1}, t\right) \\
\geq \psi\left(M\left(z_{n-1}, z_{n}, \alpha^{-1} t\right)\right)>M\left(z_{n-1}, z_{n}, \alpha^{-1} t\right) \\
\geq \ldots \geq M\left(z_{0}, z_{1}, \alpha^{-n} t\right)
\end{gathered}
$$


if $n,(n+1) \notin N^{*}, \forall t \in \boldsymbol{R}_{+}$, and:

$$
\begin{gathered}
M\left(z_{n+1}, z_{n+2}, t\right)=M\left(T z_{n}, T z_{n+1}, t\right) \\
\geq \psi\left(M\left(z_{n-1}, z_{n}, \alpha^{-1} t\right)\right)>M\left(z_{n-1}, z_{n}, \alpha^{-1} t\right) \\
\geq \ldots \geq M\left(z_{0}, z_{1}, \alpha^{-n} t\right)
\end{gathered}
$$

if $(n+1),(n+2) \in N^{*} ; \forall t \in \boldsymbol{R}_{+}$. Thus, one gets for any given $\varepsilon \in \boldsymbol{R}_{+}$, some $n_{0}=n_{0}(\varepsilon) \in \boldsymbol{Z}_{+}$, $\forall t \in \boldsymbol{R}_{+}$and all $m(\geq n), n\left(\geq n_{0}\right) \in \boldsymbol{Z}_{+}$that:

$$
\begin{gathered}
M\left(z_{n}, z_{m}, t\right) \geq \psi\left[M\left(z_{n}, z_{n+1}, t\right) * M\left(z_{n}, z_{n+1}, t\right) * \ldots * M\left(z_{m-1}, z_{m}, t\right)\right] \\
>M\left(z_{0}, z_{1}, \alpha^{-n} t\right) * M\left(z_{0}, z_{1}, \alpha^{-(n+1)} t\right) * \ldots * M\left(z_{0}, z_{1}, \alpha^{-(m+1)} t\right) \\
=\begin{array}{c}
m+1 \\
n \geq n_{0}
\end{array} M\left(x, y, \alpha^{-n} t\right) \\
\geq 1-\varepsilon
\end{gathered}
$$

from the property $T$ with $t_{n}=\alpha^{-n} t ; \forall n \in Z_{0+}$ being a $\left\{t_{n}\right\}$ s-increasing sequence for $n \geq n_{0}$ such that $t_{n_{0}}=\alpha^{-n_{0}} t \geq\left(\alpha^{-1}-1\right)^{-1}$ implies that $t_{n+1}=\alpha^{-(n+1)} t \geq t_{n}+1=\alpha^{-n} t+1$ for all $n \geq n_{0}$ and all $t \geq t_{*}=\frac{\alpha^{n_{0}}}{\alpha^{-1}-1}$ (note that $t_{*} \rightarrow 0$ as $n_{0} \rightarrow \infty$ ). Any other $s$-increasing

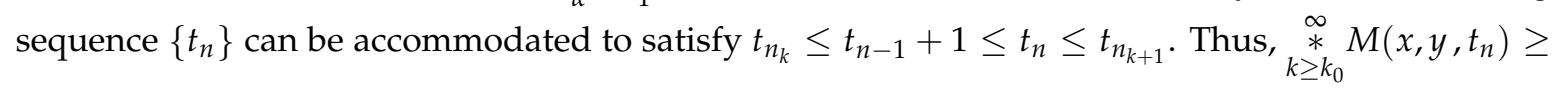
$\underset{k \geq k_{0}\left(n_{k_{0}} \geq n_{0}\right)}{\stackrel{\infty}{*}} M\left(x, y, t_{n_{k}}\right) \geq 1-\varepsilon$ by the non-Archimedean property. Then, $(X, M, *, \preceq)$ satisfies the property $\boldsymbol{T}$ since Equation (24) holds for the s-increasing sequence $\left\{\alpha^{-n} t\right\}_{n \geq n_{0}}$ and it is extendable to any other $s$-increasing sequence. Thus, one has from Equation (22) that:

$$
\lim _{k \rightarrow \infty} M\left(z_{n_{k}}, z_{n_{k}+1}, t\right)=\lim _{k \rightarrow \infty} M\left(g z_{n_{k}}, g z_{n_{k}+1}, t\right)=\lim _{k \rightarrow \infty} M\left(z_{1}, z_{0}, \alpha^{-n_{k}} t\right)=1 ; \forall t \in \boldsymbol{R}_{+}
$$

for a subsequence $\left\{z_{n_{i k}}\right\} \subseteq\left\{X_{0 i}(t)\right\}$ being defined, provided that arbitrary points $z_{0}\left(\preceq z_{1}\right) \in X_{0 i}, z_{1} \in X_{0 i}(t)$ for some given arbitrary $i \in \bar{p}$ and such that $n_{i k},\left(n_{i k}+1\right) \notin$ $N_{i}^{*}\left(N_{i}^{*} \subset N^{*}\right.$ being the set where the iteration switches from $X_{i}$ to $\left.X_{i+1}\right), \forall t \in \boldsymbol{R}_{+}$. Thus, $\left\{z_{n_{i k}}\right\}$ is Cauchy and convergent to some $x_{i}^{*} \in \operatorname{cl} X_{0 i}(t)$ since $(X, M, *, \preceq)$ is complete. For the given initial $z_{0} \in X_{0 i}(t), z_{1} \in X_{0 i}(t)$, we can always find two points $\bar{z}_{j 0} \in X_{0 j}(t), \bar{z}_{j 1}\left(\preceq \bar{z}_{j 0}\right) \in\left\{z_{n}\right\} \cap X_{0 j}(t)$, which are initial adjacent points of a subsequence $\left\{z_{n_{j k}}\right\} \subset\left\{z_{n}\right\}$ for any arbitrary $j(\neq i) \in \bar{p}$. It is found that $\left\{z_{n_{i k}}\right\}$ is Cauchy and convergent to some $x_{j}^{*} \in c l X_{0 j}(t)$. The set of $p$ limit best proximity points $x_{j}^{*} \in c l X_{0 j}(t) ; \forall j \in \bar{p}$ is unique since all the above subsequences are also totally ordered with lower-bounding and an upper-bounding ordered sequences (see Theorem 1 for similar reasoning), which converge to a unique best proximity point of a set $X_{j}$ to its adjacent set $X_{j+1}$ for each $j \in \bar{p}$. Then, for any constructed sequence $\left\{x_{n}\right\} \in \cup_{i \in \bar{p}} X_{0 i}(t)$, we have convergent subsequences to the same limit within each subset $X_{i}$, which is a unique best proximity point so that:

$$
\begin{gathered}
\lim _{k \rightarrow \infty} M\left(x_{n_{k}}, x_{n_{k}+1}, t\right)=\lim _{k \rightarrow \infty} M\left(g x_{n_{k}}, g x_{n_{k}+1}, t\right) \\
=\lim _{k \rightarrow \infty} M\left(x_{n_{j k}}, x_{j}^{*}, t\right)=\lim _{k \rightarrow \infty} M\left(g x_{n_{k}}, g x_{j}^{*}, t\right)=1 ; \forall j \in \bar{p}
\end{gathered}
$$


so that $\left\{x_{n_{j k}}\right\} \rightarrow x_{j}^{*} ; \forall j \in \bar{p}$. It is now proven that each $x_{j}^{*} \in c l X_{0 j}(t)$ is an optimal fuzzy best proximity coincidence points of the pair $(g, T)$ in $X_{j}$ for each $j \in \bar{p}$. One gets from the properties of the $t$-norms, Equation (26), and the continuity of $T: \cup_{i \in \bar{p}} X_{i} \rightarrow \cup_{i \in \bar{p}} X_{i}$ :

$$
\begin{gathered}
M\left(g x_{j}^{*}, T x_{j}^{*}, t\right) \\
\geq\left(M\left(g x_{j}^{*}, g x_{n_{j k}}, t\right) * M\left(g x_{n_{j k}} T x_{n_{j k}}, t\right)\right) * M\left(T x_{n_{j k}} T x_{j}^{*}, t\right) \\
=\left(M\left(g x_{j}^{*}, g x_{n_{j k}}, t\right) * M\left(T x_{n_{j k}} T x_{j}^{*}, t\right)\right) * M\left(g x_{n_{j k}} T x_{n_{j k}}, t\right) ; \forall k \in Z_{0+}, \forall j \in \bar{p}
\end{gathered}
$$

and:

$$
\begin{gathered}
M\left(g x_{j}^{*}, T x_{j}^{*}, t\right) \geq \underset{k \rightarrow \infty}{ } \liminf _{k \rightarrow \infty}\left(M\left(g x_{j}^{*}, g x_{n_{j k}}, t\right) * M\left(T x_{n_{j k}} T x_{j}^{*}, t\right)\right) \\
\quad \liminf _{k \rightarrow \infty} M\left(g x_{n_{j k}} T x_{n_{j k}}, t\right) \\
=(1 * 1) * \liminf _{k \rightarrow \infty} M\left(g x_{n_{j k}} T x_{n_{j k}}, t\right) \\
=\liminf _{k \rightarrow \infty}\left(g x_{n_{j k}} T x_{n_{j k}}, t\right) \\
=\lim _{k \rightarrow \infty} M\left(g x_{n_{j k}} T x_{j}^{*}, t\right) \\
=M\left(X_{j}, X_{j+1}, t\right) ; \forall j \in \bar{p}
\end{gathered}
$$

However, we can interchange the locations of $M\left(g x_{n_{j k}} T x_{j}^{*}, t\right)$ and $M\left(g x_{j}^{*}, T x_{j}^{*}, t\right)$ in Equations (27) and (28) for obtaining corresponding sets of inequalities to conclude that:

$$
\lim _{k \rightarrow \infty} M\left(g x_{n_{j k}} T x_{n_{j k}}, t\right)=M\left(g x_{j}^{*}, T x_{j}^{*}, t\right)=M\left(X_{j}, X_{j+1}, t\right) ; \forall j \in \bar{p}
$$

Thus, $x_{j}^{*}=T x_{j-1}^{*} \in c l X_{0 j}(t)$ is a unique best proximity point to $X_{j+1}$ in $X_{j}$ of $T: \cup_{i \in \bar{p}} X_{i} \rightarrow \cup_{i \in \bar{p}} X_{i}$ and it is also an optimal fuzzy best proximity coincidence point of the pair $(g, T)$ in $X_{j}$.

In the same way as Corollary 1 and under a close proof (see also Remark 3), we can get the subsequent Corollary to Theorem 2:

Corollary 3. Assume that the hypothesis of Theorem 2 holds except that, for any given initial point $z_{0} \in \cup_{i \in \bar{p}} X_{0 i}(t)$, the sequences $\left\{z_{n}\right\}$ are built so that:

(1) $z_{n+1}=T z_{n}$ for any $n \in \mathbf{Z}_{+}$if $z_{n} \in X_{0 i}(t)$ for any $i(\neq j) \in \bar{p}$ and some fixed $j \in \bar{p}$ (i.e., the proximal subsequence from each subset $X_{j}$ of $X$ to each next adjacent subset is only computed eventually at the subset $X_{0 j}(t)$, while at the remaining subsets only the cyclic self-mapping $T$ is involved);

(2) the proximal constraint Equation (3), subject to its subsequent constraints, is replaced at the subset $X_{j}$ of $X$ by the subsequent one:

$$
M\left(g z_{n_{j, k+m_{j}}+1}, T^{n_{j, k+m_{j}}+1-n_{j k}} z_{n_{j k}}, t\right)=M\left(X_{j}, X_{j+1}, t\right) ; \forall k\left(\geq k^{*}\right) \in Z_{0+,} i \in \bar{p}
$$

for some given $j \in \bar{p}$ and some subsequence $\left\{z_{n_{j k}}\right\} \subseteq X_{0 j}(t)$, for $n \in Z_{+}$with $z_{0} \in X_{i}$ for some satisfying $z_{n_{j, k+m_{j}}}=T^{n_{j, k+m_{j}(k)}-n_{j k}} z_{n_{j k}}$ with $z_{n_{j} k^{*}}, z_{n_{j} k^{*}+1} \in X_{0 j}(t)$, for some set of bounded positive integers $m_{j}(k) ; \forall k\left(\geq k^{*}\right) \in Z_{0+}$ for the given $j \in \bar{p}$ and some sequence of positive integers $\left\{n_{j k}\right\}$ being strictly increasing with $k\left(\geq k^{*}\right) \in Z_{0+}$ (i.e., the proximal subsequence at the subset $X_{0 j}$ is not necessary computed at each $k$-th cycle on the whole cyclic disposal of the $p$ subsets $X_{0 i}(t) \subseteq X_{i} \subseteq X$ for all $i \in \bar{p}$ since $m_{j}(k)$ can exceed the value $p$ for some values $\left.k\left(\geq k^{*}\right) \in \mathbf{Z}_{0+}\right)$. 
Results similar to those of Theorem 2 and Corollary 3 can be obtained by replacing the continuity assumption on $T: \cup_{i \in \bar{p}} X_{i} \rightarrow \cup_{i \in \bar{p}} X_{i}$ by the condition that $X_{i+1}$ is fuzzy approximatively compact with respect to $X_{i} ; \forall i \in \bar{p}$, that is, each sequence $\left\{T x_{n}\right\} \subset X_{i+1}$ such that $\left\{M\left(x, T x_{n}, t\right)\right\} \rightarrow M\left(x, X_{i+1}, t\right)$ for some $x \in X_{i}$ has a convergent subsequence.

Corollary 4. Theorem 2 holds "mutatis-mutandis" if $X_{i+1}$ is fuzzy and approximatively compact with respect to $X_{i} ; \forall i \in \bar{p}$ even if $T: \cup_{i \in \bar{p}} X_{i} \rightarrow \cup_{i \in \bar{p}} X_{i}$ is not everywhere continuous.

Proof. The hypothesis of Theorem 2 still holds except that $T: \cup_{i \in \bar{p}} X_{i} \rightarrow \cup_{i \in \bar{p}} X_{i}$ is not necessarily everywhere continuous while $X_{i+1}$ is fuzzy and approximately compact with respect to $X_{i} ; \forall i \in \bar{p}$. Then, the first part of the proof of Theorem 2 is still applicable while one concludes from Equation (28) that $\left\{x_{n_{j}}\right\} \rightarrow x_{j}^{*},\left\{g x_{n_{j}}\right\} \rightarrow g x_{j}^{*}$ as $n_{j} \rightarrow \infty \forall j \in \bar{p}$ so that there is a convergent subsequence $\left\{T x_{n_{j k}}\right\}\left(\subset\left\{T x_{n_{j}}\right\} \subset c l X_{0, j+1}(t)\right) \rightarrow z_{j+1}^{*}$ for some $z_{j+1}^{*} \in c l X_{0, j+1}(t)$ for each $j \in \bar{p}$ since:

$$
\begin{aligned}
M\left(g x_{j}^{*},\right. & \left.T x_{j}^{*}, t\right) \geq \liminf _{k \rightarrow \infty} M\left(g x_{n_{j k}} T x_{n_{j k}}, t\right) \\
& =\lim _{k \rightarrow \infty} M\left(g x_{n_{j k}}, z_{j+1}^{*}, t\right) \\
& =\lim _{k \rightarrow \infty} M\left(g x_{j}^{*}, T x_{n_{j k}}, t\right) \\
& =\lim _{k \rightarrow \infty} M\left(g x_{j}^{*}, z_{j+1}^{*}, t\right) \\
& =M\left(X_{j}, X_{j+1}, t\right) ; \forall j \in \bar{p}
\end{aligned}
$$

leads to:

$$
\begin{gathered}
\left(M\left(g x_{n_{j}} T x_{n_{j}}, t\right)-M\left(g x_{j}^{*}, T x_{n_{j}}, t\right)\right) \rightarrow 0, M\left(g x_{j}^{*}, T x_{n_{j}}, t\right) \rightarrow M\left(g x_{j}^{*}, X_{j+1}, t\right)=M\left(X_{j}, X_{j+1}, t\right) ; \forall j \in \bar{p}, \\
M\left(g x_{j}^{*}, z_{j+1}^{*}, t\right)=M\left(X_{j}, X_{j+1}, t\right)
\end{gathered}
$$

and $T x_{j}^{*}=z_{j+1}^{*}=x_{j+1}^{*} ; \forall j \in \bar{p}$. Assume that this is not true, i.e., $z_{j+1}^{*} \neq x_{j+1}^{*}$ for some $j \in \bar{p}$, so that:

$$
1>M\left(z_{j+1}^{*}, x_{j+1}^{*}, t\right) \geq\left(\lim _{k \rightarrow \infty} M\left(z_{j+1}^{*}, T x_{n_{j k}}, t\right) * \lim _{k, n \rightarrow \infty} M\left(T x_{n_{j k}}, T x_{n_{j}}, t\right)\right) * \lim _{n \rightarrow \infty} M\left(T x_{n_{j}}, T z_{j}^{*}, t\right)
$$

Taking limits in both sides of the above inequality as $k, n_{j}, n_{j k} \rightarrow \infty$, and since $\left\{x_{n_{j k}}\right\},\left\{x_{n}\right\} \rightarrow x_{j}^{*}$ and $\left\{T x_{n_{j k}}\right\} \rightarrow z_{j+1}^{*}$, yields the contradiction $1>(1 * 1) * 1=1$, so that $T x_{j}^{*}=z_{j+1}^{*}=x_{j+1}^{*} ; \forall j \in \bar{p}$. Then, $z_{j+1}^{*}=x_{j+1}^{*} ; \forall j \in \bar{p}$.

Corollary 5. Corollary 3 holds "mutatis-mutandis" if $X_{i+1}$ is fuzzy and approximately compact with respect to $X_{i} ; \forall i \in \bar{p}$ even if $T: \cup_{i \in \bar{p}} X_{i} \rightarrow \cup_{i \in \bar{p}} X_{i}$ is not everywhere continuous.

Theorem 2 and Corollaries 3-5 can be directly extended to a $(g, T)$ pair where the $p(\geq 2)$-cyclic mapping $T: \cup_{i \in \bar{p}} X_{i} \rightarrow \cup_{i \in \bar{p}} X_{i}$ is a continuous $p$-cyclic fuzzy order preserving proximal $\psi$-contraction of type II, the partial order being defined with respect to $\preceq$, which is now defined on the whole union of subsets of the cyclic disposal $\cup_{i \in \bar{p}} X_{i}$. This means that the constructed sequences possessing Cauchy subsequences within each subset converge to the best proximity points. Such points are simultaneously unique optimal fuzzy coincidence points of the pair $(g, T)$ that can be constructed on the whole subset $X_{i}$ but converge to the corresponding proximal subsets.

Remark 4. Note from Definition 7 and from Definition 8 that if $T: \cup_{i \in \bar{p}} X_{i} \rightarrow \cup_{i \in \bar{p}} X_{i}$ is a strong (respectively, weak) $p$-cyclic fuzzy order preserving proximal $\psi$-contraction of type II for some $\alpha \in(0,1)$ and $M\left(X_{i}, X_{i+1},.\right)$ is non-decreasing on $(0, \infty)$ then $T: \cup_{i \in \bar{p}} X_{i} \rightarrow \cup_{i \in \bar{p}} X_{i}$ is also a strong (respectively, weak) p-cyclic fuzzy order preserving proximal $\psi$-contraction of type $I$. This becomes obvious from $M\left(X_{i}, X_{i+1}, \alpha^{-1} t\right) \geq$ 
$M\left(X_{i}, X_{i+1}, t\right) ; \forall t \in \boldsymbol{R}_{0+}, \forall i \in \bar{p}$, and, respectively, from $M\left(X_{0 i}(t), X_{0, i+1}(t), \alpha^{-1} t\right) \geq$ $M\left(X_{0 i}(t), X_{0, i+1}(t), t\right) ; \forall t \in \boldsymbol{R}_{0+}, \forall i \in \bar{p}$.

Example 4. Assume that $T: \cup_{i \in \bar{p}} X_{i} \rightarrow \cup_{i \in \bar{p}} X_{i}$ is a p-cyclic fuzzy order preserving proximal $\psi$-contraction with $M(x, y, t)=\frac{\lambda t}{\lambda t+d(x, y)} ; \forall x, y \in \cup_{i \in \bar{p}} X_{i}$ (respectively, $\forall x, y \in \cup_{i \in \bar{p}} X_{0 i}(t)$ ), $\forall t \in \boldsymbol{R}_{+}$, for some real $\lambda \in \boldsymbol{R}_{+}$where $d: X \times X \rightarrow \boldsymbol{R}_{0+}$ is a metric, then, $\frac{d M(x, y, t)}{d t}=\frac{\lambda d(x, y)}{(\lambda t+d(x, y))^{2}} \geq 0, \forall x, y \in \cup_{i \in \bar{p}} X_{i}$ (respectively, $\left.\forall x, y \in \cup_{i \in \bar{p}} X_{0 i}(t)\right), \forall t \in \boldsymbol{R}_{+}$with the above inequality being strict if and only if $x \neq y$. Thus, $M\left(x, y, \alpha^{-1} t\right)>M(x, y, t)$ for any $\alpha \in(0,1)$ if and only if $x \neq y$ for all $t \in \boldsymbol{R}_{+}$. Then, $T: \cup_{i \in \bar{p}} X_{i} \rightarrow \cup_{i \in \bar{p}} X_{i}$ is also a strong (respectively, weak) p-cyclic fuzzy order preserving proximal $\psi$-contraction of type I (see also Remark 4).

Example 5. Consider the mappings $T$ and $g$ defined as in Examples 1-3. From Definition 8, Equations (22) and (23) in the proof of Theorem 2 and Example 4 with $\lambda=1$, it follows that $\lim _{n \rightarrow \infty} M\left(z_{n}, z_{n+1}, t\right) \geq \lim _{n \rightarrow \infty} M\left(z_{0}, z_{1}, \alpha^{-n} t\right)=1$ for any sequence $\left\{z_{n}\right\} \subset X_{1} \cup X_{2}$ for $i=1,2$ constructed as in the proof of Theorem 2. Thus, it follows that the $p$ subsequences $\left\{z_{\text {in }}\right\} \subset\left\{z_{n}\right\} \cap X_{i}$ (or, respectively, in $\left\{z_{i n}\right\} \subset\left\{z_{n}\right\} \cap X_{0 i}(t)$ for $i=1,2$ for the weak proximal contraction case) for each $i=1,2$ converge to unique best proximity points, which are also the unique optimal fuzzy best proximity coincidence points of the pair $(g, T)$ of $X_{i}$ at each $X_{0 i}(t) ; i=1,2$.

Remark 5. Consider the mappings $T$ and $g$ under the conditions of Theorem 3. From Equations (22) and (23) in the proof of Theorem 2 and Example 4 with $\lambda=1$, it follows that $\lim _{n \rightarrow \infty} M\left(z_{n}, z_{n+1}, t\right) \geq \lim _{n \rightarrow \infty} M\left(z_{0}, z_{1}, \alpha^{-n} t\right)=$ $M\left(z_{0}, z_{1}, \infty\right)=1$, even if the constraint $M\left(z_{n+2}, z_{n+1}, t\right) \geq \psi\left(M\left(z_{n}, z_{n+1}, \alpha^{-1} t\right)\right)$ in Definition 8 holds for all $n \in \mathbf{Z}_{0_{+}}$and only for some $t \in \boldsymbol{R}_{+}$, for any sequence $\left\{z_{n}\right\} \subset \cup_{i \in \bar{p}} X_{i}$ constructed as in the proof of Theorem 2. Thus, it follows that the $p$ subsequences $\left\{z_{i n}\right\}$ of $\left\{z_{n}\right\}$ fulfilling $\left\{z_{i n}\right\} \subset\left\{z_{n}\right\} \cap X_{i} \subset \cup_{i \in \bar{p}} X_{i}$ (or, respectively, in $\left\{z_{\text {in }}\right\} \subset\left\{z_{n}\right\} \cap X_{0 i}(t)$ for the weak proximal contraction case); $\forall i \in \bar{p}$, converge to unique best proximity points at each $X_{i}$, which are also the unique optimal fuzzy best proximity coincidence points of the pair $(g, T)$ at each $X_{i} ; \forall i \in \bar{p}$. Thus, Theorem 2 can be weakened by "ad hoc weakening" the implied part of the inequalities in Definition 8 so as to be fulfilled only for some (and not for all) $t \in \boldsymbol{R}_{+}$.

\section{Conclusions}

This paper has dealt with some properties of interest in two types of fuzzy ordered proximal contractions of cyclic self-mappings $T$, which is integrated in a pair $(g, T)$ of mappings that construct the relevant proximal sequences of interest. In particular, $g$ is supposed to be a non-contractive fuzzy self-mapping in a non-Archimedean ordered fuzzy complete metric space $(X, M, *, \preceq)$, endowed with a partial order $\preceq$ and a triangular norm $*$, while $T$ is a $p$-cyclic proximal contraction. The fuzzy set $M$ on $X \times X \times[0, \infty)$ is a fuzzy metric on the non-Archimedean fuzzy metric space $(X, M, *)$. Two types of such contractions (so-called type I and of type II) are considered. The main results obtained rely on the existence, uniqueness and limit properties for sequences to existing optimal fuzzy best proximity coincidence points for such pairs of mappings.

Acknowledgments: The first author is grateful to the Spanish Government and European Fund of Regional Development FEDER for Grant Nos. DPI2012-30651 and DPI2015-64766-R. The authors are also grateful to the referees for their useful suggestions.

Author Contributions: All authors contributed equally to the writing of this paper. All authors read and approved the final manuscript.

Conflicts of Interest: The authors declare that they have no competing interests. 


\section{References}

1. Schweizer, B.; Sklar, A. Statistical metric spaces. Pac. J. Math. 1960, 10, 314-334. [CrossRef]

2. George, A.; Veeramani, P. On some results of analysis for fuzzy metric spaces. Fuzzy Sets Syst. 1997, 90, 365-368. [CrossRef]

3. George, A.; Veeramani, P. On some results in fuzzy metric spaces. Fuzzy Sets Syst. 1994, 64, $395-399$. [CrossRef]

4. Zadeh, L.A. Fuzzy sets. Inf. Control 1965, 8, 103-112. [CrossRef]

5. Grabiec, M. Fixed points in fuzzy metric spaces. Fuzzy Sets Syst. 1983, 27, 385-389. [CrossRef]

6. Heilpern, S. Fuzzy mappings and fixed point theorem. J. Math. Anal. Appl. 1981, 83, 566-569. [CrossRef]

7. Vetro, C.; Salimi, P. Best proximity point results in non-Archimedean fuzzy metric spaces. Fuzzy Inf. Eng. 2013, 5, 417-429. [CrossRef]

8. Gregori, V.; Sapena, A. On fixed-point theorems in fuzzy metric spaces. Kybernetica 1975, 11, $326-334$. [CrossRef]

9. Sadiq Basha, S. Common best proximity points: Global minimization of multi-objective functions. J. Glob. Optim. 2012, 54, 367-373. [CrossRef]

10. Sadiq Basha, S. Best proximity points: Optimal fuzzy solutions. J. Optim. Fuzzy Theory Appl. 2011, 151, 210-216. [CrossRef]

11. Sadiq Basha, S. Best proximity point theorems on partially ordered sets. Optim. Lett. 2012, 7, 1035-1043. [CrossRef]

12. Mongkolkeha, C.; Cho, Y.J.; Kumam, P. Best proximity points for generalized proximal contraction mappings in metric spaces with partial orders. J. Inequal. Appl. 2013, 2013, 94. [CrossRef]

13. Vetro, C. Best proximity points: Convergence and existence theorems for $p$-cyclic mappings. Nonlinear Anal. Theory Methods Appl. 2010, 73, 2283-2291. [CrossRef]

14. Chitra, A.; Subrahmanyam, P.V. Fuzzy sets and fixed points. J. Math. Anal. Appl. 1987, 124, 584-590. [CrossRef]

15. Azam, A.; Beg, I. Common fuzzy fixed points for fuzzy mappings. Fixed Point Theory Appl. 2013, $2013,14$. [CrossRef]

16. Cho, Y.J.; Pathak, H.K.; Kang, S.M.; Jung, J.S. Common fixed points of compatible mappings of type $(\beta)$ on fuzzy metric spaces. Fuzzy Sets Syst. 1998, 93, 99-111. [CrossRef]

17. Abbas, M.; Altun, I.; Gopal, D. Common fixed point theorems for non compatible mappings in fuzzy metric spaces. Bull. Math. Anal. Appl. 2009, 1, 47-56.

18. Phiangsungnoen, S.; Sintunavarat, W.; Kumam, P. Common $\alpha$-fuzzy fixed point theorems for fuzzy mappings via $\beta_{F}$-admissible pair. J. Intell. Fuzzy Syst. 2014, 27, 2463-2472.

19. De la Sen, M.; Agarwal, R.P.; Ibeas, A. Results on proximal and generalized weak proximal contractions including the case of iteration-dependent range sets. Fixed Point Theory Appl. 2014, 2014, 169. [CrossRef]

20. Gabeleh, M. Best proximity point theorems via proximal non self-mapping. J. Optim. Theory Appl. 2015, 164, 565-576. [CrossRef]

21. De la Sen, M.; Roldan, A.; Agarwal, R.P. On contractive cyclic fuzzy maps in metric spaces and some related results on fuzzy best proximity points and fuzzy fixed points. Fixed Point Theory Appl. 2015, 2015, 103. [CrossRef]

22. Rashid, M.; Mehmood, N.; Azam, A.; Radenović, S. Fuzzy fixed point theorems in ordered cone metric spaces. Filomat 2015, 29, 887-896.

23. Chauan, S.; Shatanawi, W.; Kumar, S.; Radenović, S. Existence and uniquenmess of fixed points in modified intuitionistic fuzzy metric spaces. J. Nonlinear Sci. Appl. 2017, 7, 28-41.

24. Chauan, S.; Radenović, S.; Imdad, M.; Vetro, C. Some integral type fixed point theorems in non-Archimedean Menger PM-spaces with common property (EA) and applications of functional equations in dynamic programming. Rev. Real Acad. Cienc. Exact. Fís. Nat. Ser. A Mat. (RACSAM) 2014, 108, 795-810. [CrossRef]

25. Chauan, S.; Radenović, S.; Bhatnagar, S. Common fixed point theorems for weakly compatible mappings in fuzzy metric spaces. Mathematiche 2013, 68, 87-98.

26. Rezapour, S.; Derafshpour, M.; Shahzad, N. On the existence of best proximity points of cyclic contractions. Adv. Dyn. Syst. Appl. 2011, 6, 33-40. 
27. Al-Thagafi, M.A.; Shahzad, N. Convergence and existence results for best proximity points. Nonlinear Anal. Theory Methods Appl. 2009, 70, 3665-3671. [CrossRef]

28. Derafshpour, M.; Rezapour, S.; Shahzad, N. Best proximity points of cyclic $\varphi$-contractions on reflexive Banach spaces. Fixed Point Theory Appl. 2010, 2010, 946178.

29. De la Sen, M.; Karapinar, E. Some results on best proximity points of cyclic contractions in probabilistic metric spaces. J. Funct. Spaces 2015, 2015, 470574. [CrossRef]

30. De la Sen, M.; Ibeas, A. On the global stability of an iterative scheme in a probabilistic Menger space. J. Inequal. Appl. 2015, 2015, 243. [CrossRef]

31. Khojasteh, F.; Shukla, S.; Radenović, S. A new approach to the study of fixed point theory for simulation functions. Filomat 2015, 29, 1189-1194. [CrossRef]

32. De la Sen, M.; Ibeas, A. Properties of convergence of a class of iterative processes generated by sequences of self-mappings with applications to switched Systems. J. Inequal. Appl. 2014, 2014, 498. [CrossRef]

33. De la Sen, M.; Alonso-Quesada, S.; Ibeas, A. On the asymptotic hyperstability of switched systems under integral-type feedback regulation Popovian constraints. IMA J. Math. Control Inf. 2015, 32, 359-386. [CrossRef]

34. De la Sen, M.; Agarwal, R.P.; Ibeas, A.; Alonso-Quesada, S. On a generalized time-varying SEIR epidemic model with mixed point and distributed time-varying delays and combined regular and impulsive vaccination controls. Adv. Differ. Equ. 2010, 2010, 281612. [CrossRef]

35. De la Sen, M. On the robust adaptive stabilization of a class of nominally stable first-order systems. IEEE Trans. Autom. Control, 1986, 44, 597-602. [CrossRef]

36. Marchenko, V.M. Hybrid discrete-continuous systems with control: II. State-space method. Differ. Equ. 2015, 51, 54-68. [CrossRef]

37. Marchenko, V.M. Hybrid discrete-continuous control systems: I. Representation of solutions. Differ. Equ. 2015, 50, 1526-1540. [CrossRef]

38. Abbas, M.; Saleem, N.; de la Sen, M. Optimal coincidence point results in partially ordered non-Archimedean fuzzy metric spaces. Fixed Point Theory Appl. 2016, 2016, 534. [CrossRef]

39. Saleem, N.; Ali, B.; Abbas, M.; Raza, Z. Fixed points of Suzuki type generalized multivalued mappings in fuzzy metric space with applications. Fixed Point Theory Appl. 2015, 2015, 36. [CrossRef]

40. Shehu, Y.; Cai, G.; Iyiola, O.S. Iterative approximation of solutions for proximal split feasibility problems. Fixed Point Theory Appl. 2015, 2015, 123. [CrossRef]

41. Cai, G.; Shehu, Y.; Iyiola, O.S. Iterative algorithms for solving variational inequalities and fixed point problems for asymptotically nonexpansive mappings in Banach spaces. Numer. Algorithms 2016, 73, 869-906. [CrossRef]

42. Gaba, Y.U.; Iyiola, O.S. Advances in the study of metric type spaces. Appl. Math. Sci. 2015, 9, 4179-4190. [CrossRef]

43. Cai, G.; Shehu, Y.; Iyiola, O.S. Viscosity iterative algorithms for fixed point problems of asymptotically nonexpansive mappings in the intermediate sense and variational inequality problems in Banach spaces. Numer. Algorithms 2017. [CrossRef]

(C) 2017 by the authors. Licensee MDPI, Basel, Switzerland. This article is an open access article distributed under the terms and conditions of the Creative Commons Attribution (CC BY) license (http:/ / creativecommons.org/licenses/by/4.0/). 